\title{
END INVARIANTS FOR SL(2, C) CHARACTERS OF THE ONE-HOLED TORUS
}

\author{
SER PEOW TAN, YAN LOI WONG, AND YING ZHANG
}

\begin{abstract}
We define and study the set $\mathcal{E}(\rho)$ of end invariants of a $\operatorname{SL}(2, \mathbb{C})$ character $\rho$ of the one-holed torus $T$. We show that the set $\mathcal{E}(\rho)$ is the entire projective lamination space $\mathscr{P} \mathscr{L}$ of $T$ if and only if (i) $\rho$ corresponds to the dihedral representation, or (ii) $\rho$ is real and corresponds to a $\mathrm{SU}(2)$ representation; and that otherwise, $\mathcal{E}(\rho)$ is closed and has empty interior in $\mathscr{P} \mathscr{L}$. For real characters $\rho$, we give a complete classification of $\mathcal{E}(\rho)$, and show that $\mathcal{E}(\rho)$ has either 0,1 or infinitely many elements, and in the last case, $\mathcal{E}(\rho)$ is either a Cantor subset of $\mathscr{P} \mathscr{L}$ or is $\mathscr{P} \mathscr{L}$ itself. We also give a similar classification for "imaginary" characters where the trace of the commutator is less than 2. Finally, we show that for discrete characters (not corresponding to dihedral or $\mathrm{SU}(2)$ representations), $\mathcal{E}(\rho)$ is a Cantor subset of $\mathscr{P} \mathscr{L}$ if it contains at least three elements.
\end{abstract}

\section{IntroduCtion AND STATEMENT OF RESUltS}

Let $T$ be the one-holed torus, and $\pi$ its fundamental group which is free on two generators $X, Y$. The $\mathrm{SL}(2, \mathbb{C})$ character variety of $T$ is the set $\mathcal{X}$ of equivalence classes of representations $\rho: \pi \mapsto \mathrm{SL}(2, \mathbb{C})$, where the equivalence classes are obtained by taking the closure of the orbits under conjugation by $\operatorname{SL}(2, \mathbb{C})$. In this paper we define and study the set of end invariants associated to the $\operatorname{SL}(2, \mathbb{C})$ characters of the one-holed torus. To simplify the exposition, by abuse of notation, we use $\rho$ instead of $[\rho]$ to denote the characters in $\mathcal{X}$ in the rest of the paper, there should be no confusion, as we will be mostly interested in the trace function which is invariant under conjugation.

Let $\mathscr{P} \mathscr{L}$ be the projective lamination space of $T$ and $\mathscr{C} \subset \mathscr{P} \mathscr{L}$ the set of (free homotopy classes of) essential simple closed curves on $T$.

Definition 1.1. (End invariants) An element $X \in \mathscr{P} \mathscr{L}$ is an end invariant of the character $\rho$ if there exists $K>0$ and a sequence of distinct elements $X_{n} \in \mathscr{C}$ such that $X_{n} \rightarrow X$ and $\left|\operatorname{tr} \rho\left(X_{n}\right)\right|<K$ for all $n$.

Denote by $\mathcal{E}(\rho)$ the set of end invariants of $\rho$. We show that this is a closed subset of the projective lamination space $\mathscr{P} \mathscr{L}$ of $T$ (Proposition 4.4); classify the characters $\rho$ for which $\mathcal{E}(\rho)=\mathscr{P} \mathscr{L}$ and show that otherwise $\mathcal{E}(\rho)$ has empty interior in $\mathscr{P} \mathscr{L}$ (Theorem 1.2); classify characters $\rho$ for which $\mathcal{E}(\rho)=\emptyset$ (Theorem 1.3); and

2000 Mathematics Subject Classification. 57M05; 30F60; 20H10; 37F30.

Key words and phrases. one-holed torus, end invariants, $\mathrm{SL}(2, \mathbb{C})$ characters, mapping class group, simple closed curves, projective lamination, Cantor set.

The authors are partially supported by the National University of Singapore academic research grant R-146-000-056-112. The third author is also partially supported by the National Key Basic Research Fund (China) G1999075104. 
classify the possible structure of $\mathcal{E}(\rho)$ for reducible, real, imaginary and discrete characters (Theorems 1.4 1.5 1.6 and 1.7).

The set $\mathcal{E}(\rho)$ gives information about the dynamics of the action of the mapping class group $\Gamma$ of $T$ on the character $\rho$, and is closely related to the study of Kleinian groups, dynamical systems, see for example [5] or [8], and also certain problems in mathematical physics, see [8].

The character variety $\mathcal{X}$ stratifies into relative character varieties: for $\kappa \in \mathbb{C}$, the $\kappa$-relative character variety is the set of equivalence classes $\rho$ such that

$$
\operatorname{tr} \rho\left(X Y X^{-1} Y^{-1}\right)=\kappa
$$

for one (and hence any) pair of generators $X, Y$ of $\pi$. Denote by $\mathcal{X}_{\kappa}$ the $\kappa$-relative character variety. By classical results of Fricke (see for example [4 or [1]), we have the following identifications:

$$
\mathcal{X} \cong \mathbb{C}^{3}, \quad \mathcal{X}_{\kappa} \cong\left\{(x, y, z) \in \mathbb{C}^{3} \mid x^{2}+y^{2}+z^{2}-x y z-2=\kappa\right\},
$$

with the identification given by

$$
\iota: \rho \mapsto(x, y, z):=(\operatorname{tr} \rho(X), \operatorname{tr} \rho(Y), \operatorname{tr} \rho(X Y)),
$$

where $X, Y$ is a fixed pair of generators of $\pi$. The topology on $\mathcal{X}$ and $\mathcal{X}_{\kappa}$ will be that induced by the above identifications.

A character $\rho \in \mathcal{X}_{\kappa}$ such that $\iota(\rho)$ is a permutation of the triple $(0,0, \pm \sqrt{\kappa+2})$ is called a dihedral character, the image is generated by two elliptics of order 2 and contains a cyclic subgroup of index 2. A character is said to be real if $\iota(\rho)=$ $(x, y, z) \in \mathbb{R}^{3}$, and imaginary if two of the entries are purely imaginary and the third real. We adopt the convention that for imaginary characters, at least two of the entries are non-zero, so that the dihedral characters are not imaginary; this will simplify statements of results later. Real characters correspond to $\mathrm{SL}(2, \mathbb{R})$ or $\mathrm{SU}(2)$ representations while imaginary characters correspond to $\mathrm{GL}(2, \mathbb{R})$ representations (see [2], 3] and [5]).

The case $\kappa=-2$ corresponds to the so called type-preserving representations and has been extensively studied in the context of Kleinian groups, see for example [1], [6], [7].

The case $\kappa=2$ corresponds to the reducible representations and is also somewhat special, it is convenient to treat this case separately, see Theorem 1.4 and $\$ 5$ Indeed, apart from Theorems 1.2 1.4 and 5 the reader should consider all other results and sections to exclude the reducible case, to avoid unnecessary complications with exceptions caused by this case.

The mapping class group $\Gamma:=\pi_{0}\left(\mathrm{Homeo}^{+}(T)\right) \cong \mathrm{SL}(2, \mathbb{Z})$ acts on $\mathcal{X}$ and $\mathcal{X}_{\kappa}$ respectively, where we consider homeomorphisms of $T$ which fix pointwise a neighborhood of the boundary. We are interested in the large scale behavior of this action, particularly in the case where the action is not proper; this is reflected in the set of end invariants of $\rho$. The dynamics of the action was classified for real $\mathrm{SL}(2)$ characters by Goldman in [3, and a partial classification was given for imaginary characters by Stantchev in his PhD thesis in Maryland, see Goldman and Stantchev [5].

The set $\mathscr{C}$ of free homotopy classes of essential simple closed curves in $T$ can be thought of as the set of vertices of the pants graph $\mathscr{C}(T)$, where two vertices $X, Y \in$ 
$\mathscr{C}$ are connected by an edge if and only if $X, Y$ have geometric intersection number one in $T . \mathscr{C}(T)$ can be realized as the completion of the Farey triangulation $\mathcal{F}$ of the hyperbolic plane $\mathbb{H}^{2}$. In this way, $\mathscr{C}$ is naturally identified with $\mathbb{Q}:=\mathbb{Q} \cup\{\infty\}$, and the projective lamination space $\mathscr{P} \mathscr{L}$ of $T$ is identified with the projective real line $\hat{\mathbb{R}}:=\mathbb{R} \cup\{\infty\}$, the boundary of the hyperbolic plane $\mathbb{H}^{2}$. The mapping class group $\Gamma$ acts on these sets and $\mathscr{C}(T)$ in a natural way, this action is realized via the isomorphism of $\Gamma$ with $\operatorname{SL}(2, \mathbb{Z})$, which acts on the upper half-plane as a model of $\mathbb{H}^{2}$.

We now give the exact statements of our results. The first result describes all characters $\rho$ for which $\mathcal{E}(\rho)=\mathscr{P} \mathscr{L}$, and shows that otherwise, $\mathcal{E}(\rho)$ has empty interior.

Theorem 1.2. The set of end invariants $\mathcal{E}(\rho)$ is equal to $\mathscr{P} \mathscr{L}$ if and only if (i) $\rho$ is dihedral; or (ii) $\rho$ corresponds to a $\mathrm{SU}(2)$ representation. Furthermore, if $\mathcal{E}(\rho) \neq \mathscr{P} \mathscr{L}$, then $\mathcal{E}(\rho)$ has empty interior in $\mathscr{P} \mathscr{L}$.

The above can be thought of as the opposite extreme of the following theorem, characterizing the characters for which $\mathcal{E}(\rho)$ is empty, which is a consequence of results in [1] (Theorem 2), 11] (Theorem 2.3, Proposition 2.4) and 10] (Theorem 1.6); we will give a sketch of the proof in

Theorem 1.3. (Bowditch, Tan-Wong-Zhang) The set of end invariants $\mathcal{E}(\rho)$ is empty if and only if $\rho$ satisfies

(i) $\operatorname{tr} \rho(X) \notin(-2,2)$ for all $X \in \mathscr{C}$;

(ii) $|\operatorname{tr} \rho(X)| \leq 2$ for only finitely many (possibly no) $X \in \mathscr{C}$.

We call conditions (i) and (ii) in Theorem 1.3 the extended BQ-conditions.

The reducible characters $(\kappa=2)$ are somewhat special; the following result classifies $\mathcal{E}(\rho)$ for such characters.

Theorem 1.4. (End invariants for reducible characters) For $\rho \in \mathcal{X}_{2}, \mathcal{E}(\rho)=\left\{X_{0}\right\}$ or $\mathscr{P} \mathscr{L}$. Furthermore, in the first case, if $X_{0} \in \mathscr{C}$, then $\operatorname{tr} \rho\left(X_{0}\right) \in[-2,2]$ and $\operatorname{tr} \rho(X) \notin[-2,2]$ for all $X \in \mathscr{C} \backslash\left\{X_{0}\right\}$, while if $X_{0} \notin \mathscr{C}$, then $\operatorname{tr} \rho(X) \notin[-2,2]$ for all $X \in \mathscr{C}$; and in the second case, $\operatorname{tr} \rho(X) \in[-2,2]$ for all $X \in \mathscr{C}$.

Note that in particular, $\mathcal{E}(\rho)$ is never empty in this case, so that a reducible character never satisfies the extended BQ-conditions.

Denote by $\mathcal{X}^{\mathbb{R}}$ and $\mathcal{X}_{\kappa}^{\mathbb{R}}$ the real $\mathrm{SL}(2)$ character variety and relative character varieties respectively. We have the following classification of $\mathcal{E}(\rho)$ for $\rho \in \mathcal{X}_{\kappa}^{\mathbb{R}}$, together with the description of the corresponding $\rho$; we exclude the case $\kappa=2$ which was covered in the preceding theorem.

Theorem 1.5. (End invariants for real characters) Suppose $\rho \in \mathcal{X}_{\kappa}^{\mathbb{R}}$, with $\kappa \neq 2$. Then exactly one of the following must hold:

(a) $\mathcal{E}(\rho)=\emptyset$, and $\rho$ satisfies the extended BQ-conditions.

(b) $\mathcal{E}(\rho)=\{\hat{X}\}$ where $\hat{X} \in \mathscr{C}, \rho$ is a $\mathrm{SL}(2, \mathbb{R})$ representation, $\operatorname{tr} \rho(\hat{X}) \in(-2,2)$, and $\operatorname{tr} \rho(X) \notin(-2,2)$ for all $X \in \mathscr{C} \backslash\{\hat{X}\}$.

(c) $\mathcal{E}(\rho)$ is a Cantor subset of $\mathscr{P} \mathscr{L}, \rho$ is a $\mathrm{SL}(2, \mathbb{R})$ representation, $\operatorname{tr} \rho(X) \in$ $(-2,2)$ for at least two distinct $X \in \mathscr{C}$, and $\operatorname{tr} \rho(Y) \notin(-2,2) \cup\{ \pm \sqrt{\kappa+2}\}$ for some element $Y \in \mathscr{C}$.

(d) $\mathcal{E}(\rho)=\mathscr{P} \mathscr{L}$, and $\rho$ satisfies the conditions of Theorem 1.2, that is, $\rho$ is the dihedral representation or a $\mathrm{SU}(2)$ representation. 
Furthermore, case (a) occurs only when $\kappa \in(-\infty, 2) \cup[18, \infty)$; case (b) when $\kappa \in[6, \infty)$; case (c) when $\kappa \in(2, \infty)$; and case (d) when $\kappa \in[-2,2) \cup(2, \infty]$.

For $\rho \in \mathcal{X}^{\mathbb{R}}$, Theorem [1.5 implies that if $\mathcal{E}(\rho)$ has more than one element, then $\mathcal{E}(\rho)$ is either a Cantor set or all of $\mathscr{P} \mathscr{L}$. Furthermore, if $\mathcal{E}(\rho)$ has only one element $X$ (and $\kappa \neq 2$ ), then $X$ is rational, that is, corresponds to a simple closed curve. These results are not true for general complex characters, for example, punctured torus groups with two geometrically infinite ends have two end invariants, and those with one geometrically infinite end have an end invariant which is irrational. Note also that condition (ii) of the extended BQ-conditions follows from condition (i) in the case of real characters with $\kappa \neq 2$; this will follow from the proof of the theorem.

There is also an intriguing connection between the end invariants of real characters and the dynamical spectrum of the almost periodic Schrödinger operator; see [1] and 8] for details. Roughly speaking, consider a one (real) parameter family of characters $\rho_{E} \in \mathcal{X}_{\kappa}^{\mathbb{R}}$ (parametrized by the energy $E \in \mathbb{R}$ ) such that $\iota\left(\rho_{E}\right)=\left(2, E-V_{0}, E-V_{1}\right)$, where $V_{0} \neq V_{1}$ are fixed constants and $\kappa=2+\left(V_{0}-V_{1}\right)^{2}$; and an irrational element $\lambda \in \mathscr{P} \mathscr{L} \backslash \mathscr{C}$. Then the set of values of $E$ for which $\lambda \in \mathcal{E}\left(\rho_{E}\right)$ corresponds to the dynamical spectrum of a discrete almost periodic Schrödinger operator, and the conjecture is that this set is always a Cantor set.

Denote by $\mathcal{X}^{I}$ and $\mathcal{X}_{\kappa}^{I}$ the imaginary character variety and relative character varieties respectively. Recall that dihedral characters are not in $\mathcal{X}^{I}$ by our convention. Note that $\kappa \in \mathbb{R}$ in this case. We classify $\mathcal{E}(\rho)$ for $\kappa<2$ :

Theorem 1.6. (End invariants for imaginary characters)

(i) $\kappa=-2:$ For $\rho \in \mathcal{X}_{-2}^{I}, \mathcal{E}(\rho)$ is either a Cantor subset of $\mathscr{P} \mathscr{L}$, or consists of a single element $X$ in $\mathscr{C}$. In the latter case, $\operatorname{tr} \rho(X)=0$ and $\rho$ is equivalent under the action of the modular group $\Gamma$ to a character corresponding to the triple $(0, x, i x)$ where $x \in \mathbb{R}$ satisfies $|x| \geq 2$.

(ii) $-14<\kappa<2$ : For $\rho \in \mathcal{X}_{\kappa}^{I}, \mathcal{E}(\rho)$ is either a Cantor subset of $\mathscr{P} \mathscr{L}$, or consists of a single element $X$ in $\mathscr{C}$.

(iii) $\kappa \leq-14:$ For $\rho \in \mathcal{X}_{\kappa}^{I}, \mathcal{E}(\rho)$ is a Cantor subset of $\mathscr{P} \mathscr{L}$; consists of a single element $X$ in $\mathscr{C}$; or is empty.

We chose in the statement of Theorem [1.6 above to emphasize the case $\kappa=-2$ since the results are somewhat sharper than for general $-14 \leq \kappa<2$, and the case is itself of independent interest.

Our final result is for discrete characters. We say a character $\rho$ is discrete if the set of values $\{\operatorname{tr} \rho(X) \mid X \in \mathscr{C}\}$ is a discrete subset of $\mathbb{C}$. Denote the set of discrete characters by $\mathcal{X}^{\text {disc }}$. We have:

Theorem 1.7. For $\rho \in \mathcal{X}^{\text {disc }}$, if $\mathcal{E}(\rho)$ has at least three elements and $\mathcal{E}(\rho) \neq \mathscr{P} \mathscr{L}$, then $\mathcal{E}(\rho)$ is a Cantor set.

The above can be rephrased as follows: For discrete characters not corresponding to dihedral or $\mathrm{SU}(2)$ representations, $\mathcal{E}(\rho)$ is a Cantor subset of $\mathscr{P} \mathscr{L}$ if it contains at least three elements. Note that a discrete character may also have 0,1 or 2 elements in $\mathcal{E}(\rho)$.

The definition of an end invariant given generalizes the definition of a (geometrically infinite, or degenerate) end for the case where the representation is typepreserving $(\kappa=-2)$, and discrete and faithful, which is the subject of intensive 
study in the last decade, especially in relation to Thurston's Ending Lamination Conjecture, proven by Minsky (for the punctured torus) in [7. Our definition is motivated by that given by Bowditch in [1, where end invariants were defined for type-preserving but not necessarily discrete or faithful representations. In fact, this work was very much inspired by [1] and grew out of our attempt to study and develop the thread of ideas presented in $\S 5$ of 1 ].

Note however that our definition differs slightly from that used in 11; the difference is that accidental parabolics were (isolated) end invariants there, whereas they are not in ours. This slight variation simplifies the statements of the results above. It also allows us to state the following conjecture, of which the preceding results can be regarded as supporting evidence.

Conjecture 1.8. (The dendrite conjecture) Suppose that $\mathcal{E}(\rho)$ has more than two elements. Then either $\mathcal{E}(\rho)=\mathscr{P} \mathscr{L}$ or $\mathcal{E}(\rho)$ is a Cantor subset of $\mathscr{P} \mathscr{L}$.

The above conjecture is a refinement and generalization of the suggestion by Bowditch in [1] that for a generic $\rho \in \mathcal{X}_{-2}$ not satisfying the BQ-conditions, $\mathcal{E}(\rho)$ should be a Cantor set. The "convex hull" of $\mathcal{E}(\rho)$ is a subtree of the dual tree $\Sigma$ of $\mathscr{C}(T)$; the above conjecture says that this tree should look like a dendrite, in the sense that if it has more than two ends, than there should be infinite branching at any end and all ends are not isolated. The statement would have been somewhat more complicated, with several exceptions, if accidental parabolics are considered to be end invariants.

Note that in the cases considered by Bowditch, he did not really have to worry about the case $\mathcal{E}(\rho)=\mathscr{P} \mathscr{L}$ since for $\kappa=-2$ this only occurs for the trivial dihedral character corresponding to the quaternionic representation $\rho$ with $\iota([\rho])=(0,0,0)$.

Bowditch did not produce examples of characters for which $\mathcal{E}(\rho)$ was a Cantor set. Theorem 1.5 produces many such examples for real characters with $\kappa>2$ and Theorems 1.6 and 1.7 produces many non-real examples for general $\kappa$, for example the character $\rho \in \mathcal{X}_{-2}$ with $\iota(\rho)=(0,1, i)$ has $\mathcal{E}(\rho)$ a Cantor set by either theorem. To the best of our knowledge, these are the first examples for which $\mathcal{E}(\rho)$ is known to be a Cantor set.

There is also a generalization of the Ending Lamination Conjecture for $\mathrm{SL}(2, \mathbb{C})$ characters (as Bowditch conjectured for the $\kappa=-2$ case) which can be stated as follows:

Conjecture 1.9. Suppose that $\rho, \rho^{\prime} \in \mathcal{X}_{\kappa}$ are such that $\mathcal{E}(\rho)=\mathcal{E}\left(\rho^{\prime}\right), \mathcal{E}(\rho)$ has at least two elements, and $\mathcal{E}(\rho) \neq \mathscr{P} \mathscr{L}$. Then $\rho=\rho^{\prime}$.

We end this introduction with a few words about the generalizations to arbitrary surfaces. The definition of $\mathcal{E}(\rho)$ can be extended without much difficulty. The case of the four-holed sphere is similar and the techniques given here should give similar results in that case, although the analysis is generally more difficult. In other cases, $\mathscr{P} \mathscr{L}$ is homeomorphic to the sphere $S^{n}$ for some $n \geq 2$ and a possible generalization of Theorem [1.2 is that $\mathcal{E}(\rho)$ has either full measure or measure zero. A possible generalization of Conjecture 1.8 would be that $\mathcal{E}(\rho)$ is perfect, if it contains more than two elements. However, these are just speculations and we do not have any insights into these more general cases.

The rest of the paper is organized as follows. In 2 we give the notation and basic definitions to be used in the rest of the paper. In $\$$ we state three key lemmas 
used for the proofs of the theorems. In w we prove Theorems 1.2 and 1.3 In we consider reducible characters and prove Theorem 1.4 In $\$ 6$ we prove Theorems 1.5 and 1.7 and in 97 we prove Theorem 1.6 Finally, in the Appendix, we give a brief description of the $\tau$-reduction algorithm of Goldman-Stantchev in [5] for the imaginary characters, which generalizes that used by Bowditch in [1] and which is used in a crucial way in the proof of Theorem 1.6

Acknowledgements. Part of this work was carried out while the first named author was visiting the University of Maryland, College Park, the University of Warwick, and the Tokyo Institute of Technology, he would like to thank his hosts Bill Goldman, Caroline Series and Sadayoshi Kojima and these institutions for their hospitality. He would also like to thank Rich Schwartz, Rich Brown, George Stantchev, Javier Aramayona, John Parker, Juan Souto, Brian Bowditch and especially Bill Goldman, Caroline Series, Makoto Sakuma and Greg McShane for many stimulating and useful conversations. He would also like to thank Shigeru Mizushima for help with a computer program to help visualize the characters and their end invariants.

\section{NotATION AND DEFINITIONS}

In this section we introduce the notation and definitions to be used in the rest of this paper. As in the introduction, let $T$ denote the one-holed torus, that is, a torus with an open disk removed. Its fundamental group $\pi$ is freely generated by two elements $X, Y$ corresponding to two simple closed curves on $T$ with intersection number one.

2.1. The (relative) character variety $\mathcal{X}\left(\right.$ resp. $\left.\mathcal{X}_{\kappa}\right)$. The $\mathrm{SL}(2, \mathbb{C})$ character variety is the set

$$
\mathcal{X}=\operatorname{Hom}(\pi, \operatorname{SL}(2, \mathbb{C})) / / \operatorname{SL}(2, \mathbb{C})
$$

where the quotient is the geometric invariant theory quotient by the conjugation action. By abuse of notation, denote by $\rho$ (instead of $[\rho]$ ) the elements of $\mathcal{X}$; we call them characters of $T$. For $\kappa \in \mathbb{C}$, the $\kappa$-relative character variety is the subset

$$
\mathcal{X}_{\kappa}=\left\{\rho \in \mathcal{X} \mid \operatorname{tr} \rho\left(X Y X^{-1} Y^{-1}\right)=\kappa\right\},
$$

where $X, Y$ are generators of $\pi$. By results of Fricke, it does not matter which pair of generators $X, Y$ are used to define $\kappa$. We have

$$
\mathcal{X} \cong \mathbb{C}^{3}, \quad \mathcal{X}_{\kappa} \cong\left\{(x, y, z) \in \mathbb{C}^{3} \mid x^{2}+y^{2}+z^{2}-x y z-2=\kappa\right\} ;
$$

the identification is given by

$$
\iota: \rho \mapsto(x, y, z):=(\operatorname{tr} \rho(X), \operatorname{tr} \rho(Y), \operatorname{tr} \rho(X Y)),
$$

where $X, Y$ is a fixed pair of free generators of $\pi$. Conversely, $\iota^{-1}(x, y, z)$ can be realized by the following representation (see [3]):

$$
\rho(X)=A:=\left(\begin{array}{cc}
x & 1 \\
-1 & 0
\end{array}\right), \quad \rho(Y)=B:=\left(\begin{array}{cc}
0 & -\zeta \\
\zeta^{-1} & y
\end{array}\right),
$$

where $\zeta+\zeta^{-1}=z$.

2.2. Topology of the (relative) character variety. The topology on $\mathcal{X}$ and $\mathcal{X}_{\kappa}$ will be that induced by the identifications defined in (10) respectively. 
2.3. Action of the mapping class group $\Gamma$. The mapping class group

$$
\Gamma:=\pi_{0}\left(\operatorname{Homeo}^{+}(T)\right) \cong \mathrm{SL}(2, \mathbb{Z})
$$

acts on $\pi$ and hence on $\mathcal{X}$; the action is given by

$$
\phi(\rho)=\rho \circ \phi^{-1},
$$

where $\phi \in \Gamma$ and $\rho \in \mathcal{X}$. The action is not effective, the kernel is generated by the elliptic involution corresponding to $-I \in \mathrm{SL}(2, \mathbb{Z})$, so the effective action is by $\operatorname{SL}(2, \mathbb{Z}) / \pm I=\operatorname{PSL}(2, \mathbb{Z})$. The quantity $\kappa=\operatorname{tr} \rho\left(X Y X^{-1} Y^{-1}\right)$ is preserved under the action of $\Gamma$ by results of Nielsen (see [3]); hence $\Gamma$ also acts on the relative varieties $\mathcal{X}_{\kappa}$. With the identification of $\mathcal{X}$ and $\mathcal{X}_{\kappa}$ with the complex varieties in (11), the action of $\Gamma$ is realized via polynomial maps on these varieties; it is generated by the cyclic permutation

$$
c:(x, y, z) \mapsto(z, x, y)
$$

and the involution

$$
\begin{gathered}
s:(x, y, z) \mapsto(y, x, x y-z) \\
\text { corresponding to }\left(\begin{array}{cc}
1 & -1 \\
1 & 0
\end{array}\right),\left(\begin{array}{cc}
0 & 1 \\
-1 & 0
\end{array}\right) \in \operatorname{PSL}(2, \mathbb{Z}) \text { respectively. }
\end{gathered}
$$

2.4. Sign change automorphisms. There is a $\mathbb{Z} / 2 \times \mathbb{Z} / 2$ action on $\mathcal{X}$ (resp. $\left.\mathcal{X}_{\kappa}\right)$ generated by simultaneously changing the signs of two of the entries of $\iota(\rho)=$ $(x, y, z)$. Two characters are equivalent under this action if and only if they correspond to lifts of the same representation of $\pi$ into $\operatorname{PSL}(2, \mathbb{C})$. The large scale behavior of the action of $\Gamma$ on $\mathcal{X}$ and the end invariants of $\rho$ are not affected by these sign change automorphisms; nonetheless, it will be convenient to use them for some local trace reduction arguments later.

2.5. The pants graph $\mathscr{C}(T)$ and the projective lamination space $\mathscr{P} \mathscr{L}$. Let $\mathscr{C}$ denote the set of free homotopy classes of essential (nontrivial, non-boundary) simple closed curves on $T$, which can be regarded as the set of vertices of the pants graph $\mathscr{C}(T)$ of $T$, where two vertices are connected by an edge if and only if the corresponding curves have geometric intersection number one. The pants graph $\mathscr{C}(T)$ can be concretely realized as the completion $\overline{\mathcal{F}}$ of the Farey tessellation $\mathcal{F}$ of the upper half-plane $\mathbb{H}^{2}$ in $\mathbb{H}^{2} \cup \hat{\mathbb{R}}$ (see Figure 1); recall that $\mathcal{F}$ is the tessellation of $\mathbb{H}^{2}$ by ideal triangles where the edges are the translates of the infinite geodesic $(0, \infty)$ by $\operatorname{PSL}(2, \mathbb{Z})$. Note that $\mathscr{C}(T)$ is not locally finite, and every vertex has infinite degree. The space $\mathscr{P} \mathscr{L}$ of projective laminations on $T$ can be regarded as the completion of $\mathscr{C}$; in this way, $\mathscr{P} \mathscr{L}$ can be identified with $\hat{\mathbb{R}}$, where the irrational points of $\mathbb{R}$ correspond to projective laminations which are not closed, and $\mathscr{C}$ is identified with $\hat{\mathbb{Q}}$, once we fix an identification of $X, Y$ and $X Y$ with $0, \infty$ and 1, where $X, Y$ are a fixed pair of generators of $\pi$. There is a natural topology on $\mathscr{P} \mathscr{L}$ induced from the topology of $\hat{\mathbb{R}}$ which agrees with the usual topology on $\mathscr{P} \mathscr{L}$ regarded as the completion of $\mathscr{C}$. There is also a natural orientation induced on $\mathscr{P} \mathscr{L}$ from this identification, where we use the usual orientation of $\hat{\mathbb{R}}$. It is also convenient to use the conformal unit disk model of the hyperbolic plane to visualize all this; in this way, $\mathscr{C}(T)$ is a triangulation of the unit disk $D$, and $\mathscr{P} \mathscr{L}$ is identified with the unit circle $S^{1} \cong \hat{\mathbb{R}}$, where the anti-clockwise direction is positive. We use upper case letters to denote elements of $\mathscr{C}$, and more generally, elements of $\mathscr{P} \mathscr{L}$. Occasionally, we will also use $\lambda$ to denote an element of $\mathscr{P} \mathscr{L} \backslash \mathscr{C}$. 
2.6. The dual trivalent tree $\Sigma$. The dual graph of $\mathcal{F}($ or $\mathscr{C}(T))$ is an infinite trivalent tree $\Sigma$. Geometrically, we may choose the vertices of $\Sigma$ as the incenters of the ideal triangles in the tessellation $\mathcal{F}$ and the edges as the geodesic arcs connecting the incenters of pairs of adjacent ideal triangles in $\mathcal{F}$, where two ideal triangles are said to be adjacent if they share a common side. $\Sigma$ is trivalent since for each ideal triangle in $\mathcal{F}$ there are exactly three others adjacent to it; see Figure 1 Note that $\Sigma$ is properly imbedded in the hyperbolic plane and all its ends form the whole ideal boundary of the hyperbolic plane. Denote by $V(\Sigma), E(\Sigma)$ the sets of vertices and edges of $\Sigma$ respectively, and we use the notation $v, e$ to represent elements of $V(\Sigma)$ and $E(\Sigma)$ respectively. A complementary region of $\Sigma$ is the closure of a connected component of the complement of $\Sigma$ in $\mathbb{H}^{2}$; we denote the set of complementary regions of $\Sigma$ by $\Omega(\Sigma)$.

2.7. Generating pairs, triples and quadruples. For $X, Y, Z, Z^{\prime} \in \mathscr{C}$ :

- The (unordered) pair $(X, Y)$, is a generating pair if $X$ and $Y$ are connected by an edge of $\mathscr{C}(T)$. We say that $X$ and $Y$ are neighbors (in $\mathscr{C}(T)$ );

- The (unordered) triple $(X, Y, Z)$ is a generating triple if $X, Y$ and $Z$ are the vertices of a triangle in $\mathscr{C}(T)$; and

- $\left(X, Y ; Z, Z^{\prime}\right)$ (where each of the first and second pair in the quadruple is unordered) is a generating quadruple if $(X, Y, Z)$ and $\left(X, Y, Z^{\prime}\right)$ are generating triples (note that the pair $Z, Z^{\prime}$ is determined uniquely by $X, Y$ ).

We shall denote the sets of generating pairs, triples and quadruples by $\mathcal{G P}, \mathcal{G} \mathcal{T}$ and $\mathcal{G Q}$ respectively.

2.8. Correspondences between various sets of objects. There are natural correspondences between the following sets, which are self-evident (see Figure 2):

$$
\begin{aligned}
& V(\Sigma) \longleftrightarrow \mathcal{G T} ; v \mapsto(X, Y, Z) \\
& E(\Sigma) \longleftrightarrow \mathcal{G P} \longleftrightarrow \mathcal{G Q} ; e \leftrightarrow(X, Y) \leftrightarrow\left(X, Y ; Z, Z^{\prime}\right) \\
& \Omega(\Sigma) \longleftrightarrow \mathscr{C} \longleftrightarrow \mathbb{Q} .
\end{aligned}
$$

In the second correspondence, $(X, Y)$ defines an edge in $\mathcal{F}$ which is dual to $e$. In the last correspondence, we will use the same letters $X, Y, Z$ to denote the elements of all three sets, namely, $\Omega(\Sigma), \mathscr{C}$ and $\widehat{\mathbb{Q}}$. Indeed, we shall use the correspondence freely, so that the same symbol $X$ may denote an element of $\Omega(\Sigma), \mathscr{C}$ or $\hat{\mathbb{Q}}$; it should be clear from the context which one we mean. We will also use the symbols $X(p / q)$ to indicate that $X \in \mathscr{C}$ (or $\Omega(\Sigma)$ ) corresponds to $p / q \in \hat{\mathbb{Q}}$.

2.9. Directed edges of $\Sigma$. Let $\vec{E}(\Sigma)$ denote the set of directed edges of $\Sigma$. Denote by $\vec{e}$ the elements of $\vec{E}(\Sigma)$, where the direction of the arrow goes from the tail to the head. We use the notation $\vec{e} \leftrightarrow\left(X, Y ; Z \rightarrow Z^{\prime}\right)$ to indicate that the directed edge $\vec{e}$ corresponds to the generating quadruple $\left(X, Y ; Z, Z^{\prime}\right)$ with the direction of the arrow pointing from $Z$ towards $Z^{\prime}$. We shall also use the orientation convention that $X, Y, Z$ are in clockwise order as points on $\hat{\mathbb{R}}$ (as the boundary of $\mathbb{H}^{2}$ ), so that if $\vec{e} \leftrightarrow\left(X, Y ; Z \rightarrow Z^{\prime}\right)$, then $-\vec{e} \leftrightarrow\left(Y, X ; Z^{\prime} \rightarrow Z\right)$ where $-\vec{e}$ is the directed edge which is directed in the opposite direction of $\vec{e}$ and has the same underlying undirected edge $e$. In other words, directed edges correspond to ordered generating pairs, and ordered generating quadruples; see Figure 2 


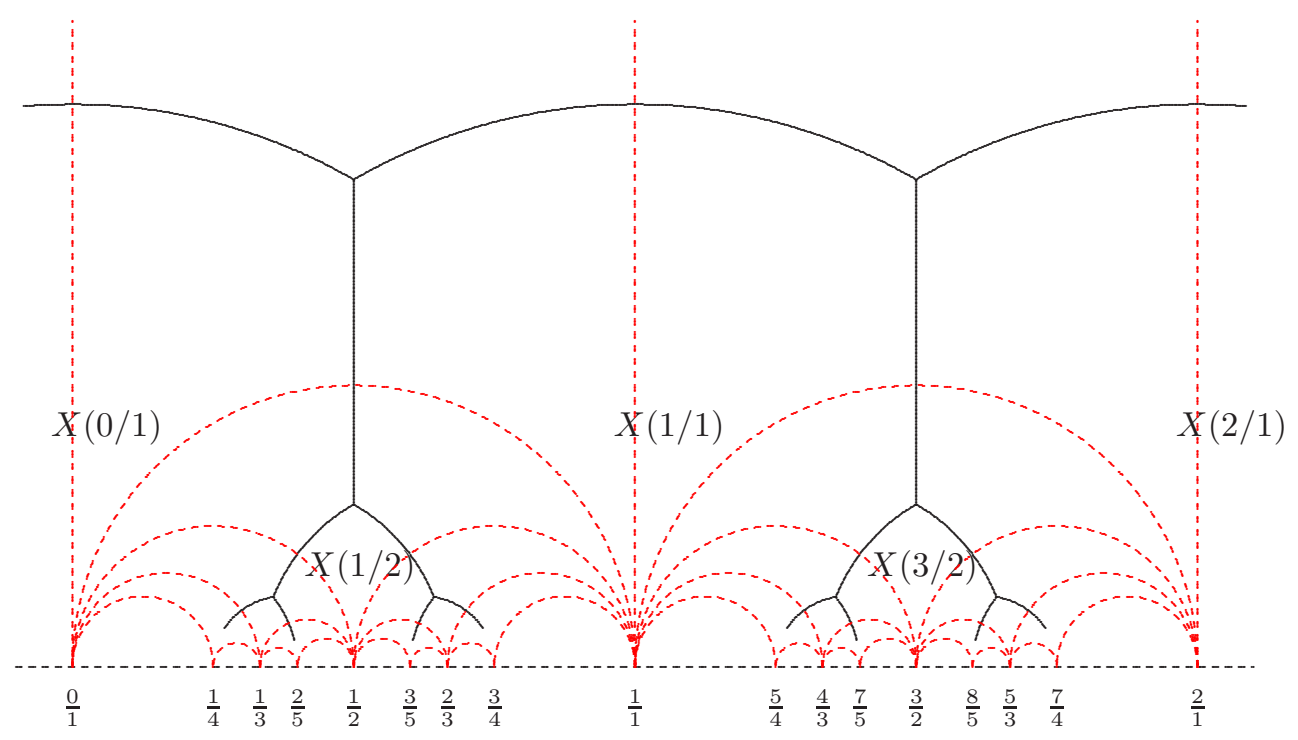

Figure 1. Farey tessellation $\mathcal{F}$ and the dual trivalent tree $\Sigma$.

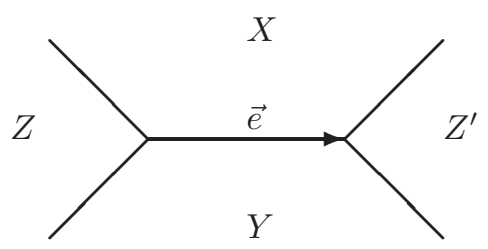

Figure 2. The directed edge $\vec{e} \leftrightarrow\left(X, Y ; Z \rightarrow Z^{\prime}\right)$

2.10. Tri-coloring of $\mathscr{C}, \Omega(\Sigma)$ and $E(\Sigma)$. The set $\mathscr{C}$ (or $\Omega(\Sigma)$ ) can be naturally partitioned into three equivalence classes, $\mathcal{R}, \mathcal{G}$ and $\mathcal{B}$ (we use the colors Red, Green and Blue to denote each class) in such a way that any generating triple $(X, Y, Z)$ contains exactly one element in each class. Similarly, $E(\Sigma)$ admits a partition into three equivalence classes $E_{r}, E_{g}$ and $E_{b}$, where if $e \leftrightarrow(X, Y)$, then $e \in E_{r}$ if $X, Y \notin \mathcal{R}$ and so on.

2.11. Subsets of $\mathscr{C}$ and $\mathscr{P} \mathscr{L}$. Denote by $[X, Y]$ the set of points in $\mathscr{P} \mathscr{L}$ in the closed interval from $X$ to $Y$, going in the anti-clockwise/positive direction, so for example $[X(0), X(1)]$ consists of all points in $\mathscr{P} \mathscr{L}$ corresponding to real numbers in the interval $[0,1]$ and $[X(1), X(0)]$ correspond to points in $\hat{\mathbb{R}}$ outside the open interval $(0,1)$. Denote similarly the half open and open subsets of $\mathscr{P} \mathscr{L}$ by $[X, Y)$, $(X, Y]$ and $(X, Y)$ (note that we use the same notation for a generating pair but it should be clear from the context if we mean a generating pair, or the corresponding open interval in $\mathscr{P} \mathscr{L}$ ). Denote by $\mathscr{C}_{[X, Y]}$ (and similarly for the other intervals) the 
intersection $\mathscr{C} \cap[X, Y]$. To each directed edge $\vec{e} \leftrightarrow\left(X, Y ; Z \rightarrow Z^{\prime}\right)$, we associate the subset of $\mathscr{C}$, called the tail of $\vec{e}$ defined by $\operatorname{tail}(\vec{e}):=\mathscr{C}_{[X, Y]}$. Note that this is the intersection of $\mathscr{C}$ with the closed interval in $\mathscr{P} \mathscr{L}$ with end points $X$ and $Y$ which contain $Z$.

2.12. The Fricke trace map induced by a character $\rho$. A character $\rho \in \mathcal{X}_{\kappa}$ induces a map

$$
\phi:=\phi_{\rho}: \mathscr{C} \rightarrow \mathbb{C}
$$

given by $\phi(X)=\operatorname{tr} \rho(X)$. Equivalently, $\phi$ can be thought of as a map from $\Omega(\Sigma)$ to $\mathbb{C}$, from the correspondence between $\mathscr{C}$ and $\Omega(\Sigma)$. We adopt the convention that the corresponding lower case letters denote the values of $\phi$, that is $\phi(X)=x$, $\phi(Y)=y, \phi(Z)=z$ and so on. This will simplify notation considerably. This map is called a Markoff map by Bowditch in 1] when $\kappa=-2$, and a generalized Markoff map by the authors in [11] for general $\kappa$ (where the map was defined from $\Omega(\Sigma)$ to $\mathbb{C})$; we call this the Fricke map or Fricke trace map in this paper. At any rate, $\phi$ satisfies the following vertex and edge relations:

$$
x^{2}+y^{2}+z^{2}-x y z-2=\kappa,
$$

for any generating triple $(X, Y, Z) \in \mathcal{G} \mathcal{T}$; and

$$
z+z^{\prime}=x y
$$

for any generating quadruple $\left(X, Y ; Z, Z^{\prime}\right) \in \mathcal{G Q}$ (recall we are using the corresponding lower case letters to denote the values of $\phi)$.

The vertex relation (7) holds for every generating triple if it holds for one by the edge relation (8), that is, it propagates along the tree $\Sigma$. These relations arise from the corresponding Fricke trace identities:

$$
\begin{gathered}
(\operatorname{tr} A)^{2}+(\operatorname{tr} B)^{2}+(\operatorname{tr} A B)^{2}-\operatorname{tr} A \operatorname{tr} B \operatorname{tr} A B-2=\operatorname{tr}[A, B] \\
\operatorname{tr} A B+\operatorname{tr} A B^{-1}=\operatorname{tr} A \operatorname{tr} B
\end{gathered}
$$

Note that the sign-change automorphism described in $\$ 2.4$ corresponds to changing the signs of $\phi(X)$ for all $X$ in two of the classes in the tri-coloring of $\mathscr{C}$ described in 2.10 while keeping the entries in the third class fixed.

2.13. The extended BQ-conditions. Let $\phi$ be a Fricke trace map and $S \subseteq \mathscr{C}$. We say that $\phi$ satisfies the extended BQ-conditions on $S$ if

(i) $\phi(X) \notin(-2,2)$ for all $X \in S$;

(ii) $|\phi(X)| \leq 2$ for only finitely many (possibly no) $X \in S$.

If $S=\mathscr{C}$, we say that $\phi$ satisfies the extended BQ-conditions. If $\mathscr{C}=S_{1} \cup \cdots \cup S_{n}$, then clearly $\phi$ satisfies the extended BQ-conditions if and only if it does on all the $S_{i}, i=1, \cdots, n$.

2.14. Dihedral characters. Note that $\iota^{-1}(0,0, \pm \sqrt{\kappa+2})$ is the representation

$$
\rho(X)=A:=\left(\begin{array}{cc}
0 & 1 \\
-1 & 0
\end{array}\right), \quad \rho(Y)=B:=\left(\begin{array}{cc}
0 & -\zeta \\
\zeta^{-1} & 0
\end{array}\right),
$$

where $\zeta+\zeta^{-1}= \pm \sqrt{\kappa+2}$. We call any character for which two of the entries of $\iota(\rho)$ are zero dihedral characters; they are generated by two order two elliptics $A$ and $B$ as above, and contain the cyclic subgroup $\left\langle\left(\begin{array}{cc}\zeta & 0 \\ 0 & \zeta^{-1}\end{array}\right)\right\rangle$ as a normal subgroup 
of index two. By (8), if $\rho$ is a dihedral character, $\phi(X)$ takes only the values 0 , $\pm \sqrt{\kappa+2}$ for all $X \in \mathscr{C}$.

2.15. Real and imaginary characters. A character $\rho$ is real if $\iota(\rho)=(x, y, z) \in$ $\mathbb{R}^{3}$. Real characters correspond to $\mathrm{SL}(2, \mathbb{R})$ or $\mathrm{SU}(2)$ representations, see [2] or 3. The real (relative) $\kappa$-character variety can be identified with the real algebraic variety

$$
\mathcal{X}_{\kappa}^{\mathbb{R}} \cong\left\{(x, y, z) \in \mathbb{R}^{3} \mid x^{2}+y^{2}+z^{2}-x y z-2=\kappa\right\} .
$$

A real character corresponds to a $\mathrm{SU}(2)$ representation if and only if for any generating triple $(X, Y, Z),-2 \leq x, y, z \leq 2$, and $\kappa \leq 2$ (see 3 ). Note that $\kappa \geq-2$ in this case. For an $\mathrm{SL}(2, \mathbb{R})$ character which is not dihedral, it is easy to see that for any $K>0$, there exists $X \in \mathscr{C}$ with $|x|>K$ (see [3]).

A character $\rho$ is imaginary if $\iota(\rho)$ is a triple with two purely imaginary entries and one real entry, and at least two entries are non-zero. If we use $i x$ and $i y$ for the imaginary entries and $z$ for the real entry, where $x, y, z \in \mathbb{R}$, then the elements of $\mathcal{X}_{\kappa}^{I}$ can be identified with the points on the real algebraic variety (taking away the points on the coordinate axes since we are assuming the character is not dihedral)

$$
\left\{(x, y, z) \in \mathbb{R}^{3} \mid-x^{2}-y^{2}+z^{2}+x y z-2=\kappa\right\} .
$$

2.16. Reducible characters. A character $\rho$ is reducible if $\kappa=2$ (see [3]). In this case, $\rho(X)$ and $\rho(Y)$ have a common fixed point, which we may assume to be $\infty$, so that $\rho$ is conjugate to an upper triangular representation. We can replace $\rho$ with its semi-simplification which is a representation by diagonal matrices with the same character, and henceforth we shall do so. Hence the representations we shall consider for reducible characters $\rho$ with $\iota(\rho)=(x, y, z)$ will be of the form

$$
\rho(X)=\left(\begin{array}{cc}
\xi & 0 \\
0 & \xi^{-1}
\end{array}\right), \quad \rho(Y)=\left(\begin{array}{cc}
\eta & 0 \\
0 & \eta^{-1}
\end{array}\right), \quad \rho(X Y)=\left(\begin{array}{cc}
\zeta & 0 \\
0 & \zeta^{-1}
\end{array}\right)
$$

where $\xi+\xi^{-1}=x, \eta+\eta^{-1}=y$, and $\zeta+\zeta^{-1}=z$.

2.17. Discrete characters. A character $\rho \in \mathcal{X}_{\kappa}$ is said to be discrete if the set $\{\phi(X) \mid X \in \mathscr{C}\}$ is a discrete subset of $\mathbb{C}$. Note that this occurs for example if $\iota(\rho)=(x, y, z)$ and $x, y$ and $z$ all lie in a discrete ring in $\mathbb{C}$, for example $\mathbb{Z}$ or $\mathbb{Z}[i]$, or more generally the ring of integers $\mathcal{O}_{d}$ of $\mathbb{Q}(\sqrt{d})$, where $d<0$ is a square-free integer.

2.18. The flow on $\Sigma$ associated to a character $\rho$. Associated to a character $\rho$ is a flow on the tree $\Sigma$, that is, a map $f:=f_{\rho}: E(\Sigma) \rightarrow \vec{E}(\Sigma)$ defined as follows: For $e \leftrightarrow\left(X, Y ; Z, Z^{\prime}\right), f(e)=\vec{e} \leftrightarrow\left(X, Y ; Z \rightarrow Z^{\prime}\right)$ if $|z| \geq\left|z^{\prime}\right|$, that is, the flow goes from the larger absolute value of $\phi$ to the smaller one along $\Sigma$. If $|z|=\left|z^{\prime}\right|$, the flow is defined arbitrarily for that edge; this ambiguity does not have any serious consequences on the subsequent developments. A vertex $v \in V(\Sigma)$ is called a sink if $f(e)$ is directed towards $v$ for all the three edges $e \in E(\Sigma)$ meeting at $v$. A finite subtree $\Sigma_{0}$ of $\Sigma$ is called an attractor for the flow if $f(e)$ is directed towards $\Sigma_{0}$ for all $e \in \Sigma \backslash \Sigma_{0}$. Note that a sink $v \leftrightarrow(X, Y, Z)$ may or may not be an attractor for the flow; however, it is if in addition $|x|,|y|,|z|>2$. 
2.19. End invariants. End invariants for a character $\rho$ are defined as in Definition 1.1 in the introduction; the set of end invariants is denoted by $\mathcal{E}(\rho)$. We shall see in 6 that there are other equivalent definitions which we will use. The relation between the end invariants and (non-)properness of the action of $\Gamma$ on $\rho$ can be described as follows. $X \in \mathscr{P} \mathscr{L}$ is an end invariant of $\rho \in \mathcal{X}_{\kappa}$ if and only if there exists a sequence $\left\{\theta_{n}\right\} \subset \Gamma \cong \operatorname{SL}(2, \mathbb{Z})$ with $\left|\operatorname{tr} \theta_{n}\right| \rightarrow \infty$ such that $\theta_{n}(\rho)$ stays in a fixed compact subset $S$ of $\mathcal{X}_{\kappa}$ (independent of $\rho$ ), and the repelling fixed points $\mu_{n}^{-} \in \mathscr{P} \mathscr{L}$ of $\theta_{n}$ approach $X$ as $n \rightarrow \infty$.

\section{BASIC RESUlts}

Much of the ensuing discussion in this paper hinges on three fairly elementary but fundamental results on quasi-convexity (Lemma 3.1), escaping orbits (Lemma 3.4) and behavior of neighbors around $X$ (Lemma 3.5) which are easy to prove but play key roles in controlling the large scale behavior of the action of $\Gamma$ on the character $\rho$, and in the proof of the theorems. They were first proved by Bowditch in 11 for $\kappa=-2$; the generalization to arbitrary $\kappa$ can be found in 11.

For the rest of this section, we fix a $\rho \in \mathcal{X}_{\kappa}$ and let the corresponding Fricke trace map be $\phi:=\phi_{\rho}$, which we take to be a map from $\Omega(\Sigma)$ to $\mathbb{C}$. We also assume $\kappa \neq 2$. Recall that we adopt the convention $\phi(X)=x, \phi(Y)=y$, and so on.

3.1. Quasi-convexity: Connectedness of $\mathscr{C}_{\phi}(K)$ for $K \geq 2$. We say that a subset $S \subset \mathscr{C}$ is connected if the subgraph spanned by $S$ in the pants graph $\mathscr{C}(T)$ is connected. For $K>0$, let $\mathscr{C}(K):=\mathscr{C}_{\phi}(K)=\{X \in \mathscr{C}|| \phi(X) \mid \leq K\}$, and we define $\Omega(K)$ similarly. We then have

Lemma 3.1. (Quasi-connectivity) For all $K \geq 2, \mathscr{C}(K)$ (equivalently, $\Omega(K)$ ) is connected.

Lemma 3.1 can be deduced easily from the following results.

Proposition 3.2. Suppose that $K \geq 2$ and $\left(X, Y ; Z, Z^{\prime}\right)$ is a generating quadruple such that $Z, Z^{\prime} \in \mathscr{C}(K)$. Then either $X$ or $Y$ (or both) is in $\mathscr{C}(K)$.

Proof. This follows directly from the edge relation (8).

Proposition 3.3. Suppose $X, Y, Z \in \Omega(\Sigma)$ meet at a vertex $v \in V(\Sigma)$, and that the arrows on the edges $X \cap Y$ and $X \cap Z$ arising from the flow $f_{\rho}$ both point away from $v$. Then either $|x| \leq 2$, or $y=z=0$.

Proof. Let $Z^{\prime}$ and $Y^{\prime}$ be the regions opposite to $Z$ and $Y$ respectively, from the vertex $v$. By the assumption of the direction of the arrow on the edge $X \cap Y$, we have $2|z| \geq|z|+\left|z^{\prime}\right| \geq\left|z+z^{\prime}\right|=|x y|$. Similarly, $2|y| \geq|x z|$. Adding, we get $2(|z|+|y|) \geq|x|(|y|+|z|)$ from which the conclusion follows.

Sketch of proof of Lemma 3.1 We prove connectedness of $\Omega(K)$. Suppose that this is not connected. Take a minimal path in $\Sigma$ connecting two of the components. If this path consists of only one edge in $\Sigma$, we get a contradiction by Proposition 3.2 If it consists of more than one edge, then by the construction, the flow at edges on the two ends of the path point outwards, so that there is a vertex inside this path where two of the arrows are pointing outwards, and we get a contradiction by Proposition 3.3 


\subsection{Escaping orbits.}

Lemma 3.4. (Escaping orbits) Suppose that $\left\{\vec{e}_{n}\right\}, n \in \mathbb{N}$ is an infinite directed path in $\Sigma$ with the head of $\vec{e}_{n}$ equal to the tail of $\vec{e}_{n+1}$, and such that $f_{\rho}\left(e_{n}\right)=$ $\vec{e}_{n}$. Furthermore, suppose that $\left\{\vec{e}_{n}\right\}$ does not limit to a rational point of $\mathscr{P} \mathscr{L}$. Then there exists infinitely many $X \in \mathscr{C}(2)$ such that the path $\left\{\vec{e}_{n}\right\}$ intersects the boundary of the corresponding complementary regions $X \in \Omega(\Sigma)$.

Proof. See [1] for the case $\kappa=-2$ and [1] for the extension to the general case where $\kappa \neq 2$.

3.3. Neighbors around $X$. For each $X \in \mathscr{C}$, let $Y_{n}, n \in \mathbb{Z}$ be the consecutive neighbors of $X$, so that $\left(X, Y_{n}, Y_{n+1}\right) \in \mathcal{G T}$ is a generating triple for all $n$. For example, if $X$ corresponds to $\infty \in \hat{\mathbb{Q}}$, then we can take $Y_{n}$ to correspond to $n \in \mathbb{Z}$. Let $x=\lambda+\lambda^{-1}$ where $|\lambda| \geq 1$. Note that $|\lambda|=1$ if and only if $x \in[-2,2] \subset \mathbb{R}$. If $x=2$, then from the vertex relation (7) and edge relation (8), $y_{n+1}=y_{n} \pm \sqrt{\kappa-2}$, and $y_{n+1}-y_{n}=y_{n}-y_{n-1}$, hence the \pm sign is constant in $n$. Similarly, if $x=-2$, then $y_{n+1}=-y_{n} \pm \sqrt{\kappa-2}$, but this time, $y_{n+1}+y_{n}=-\left(y_{n}+y_{n-1}\right)$, hence the \pm sign alternates in $n$. If $x= \pm \sqrt{\kappa+2}$, then $y_{n+1}=\lambda^{ \pm 1} y_{n}$ where the \pm sign is constant in $n$. If $x \notin\{ \pm 2, \pm \sqrt{\kappa+2}\}$ then there are (non-zero) constants $A, B \in \mathbb{C} \backslash\{0\}$ with $A B=\left(x^{2}-\kappa-2\right) /\left(x^{2}-4\right)$ such that $y_{n}=A \lambda^{n}+B \lambda^{-n}$. Hence we deduce that the following holds. (This is Corollary 3.3 in [1 in the case $\kappa=-2$.)

Lemma 3.5. (Neighbors of $X$ ) Suppose that $X \in \mathscr{C}$ has consecutive neighbors $Y_{n}$, $n \in \mathbb{Z}$. Let $\rho \in \mathcal{X}_{\kappa}, \kappa \neq 2$, with corresponding Fricke trace map $\phi$.

(a) If $x \notin[-2,2] \cup\{ \pm \sqrt{\kappa+2}\}$, then $\left|y_{n}\right|$ grows exponentially as $n \rightarrow \infty$ and as $n \rightarrow-\infty$.

(b) If $x \in(-2,2)$, then $\left|y_{n}\right|$ remains bounded. The values of the neighbors $y_{n}$ are periodic if $x=2 \cos \frac{p}{q} \pi$ for some $p / q \in \mathbb{Q}$, and quasi-periodic otherwise, that is, for any $n \in \mathbb{Z}$ and $\epsilon>0$, there exists infinitely many indices $n_{k}$ with $\left|y_{n}-y_{n_{k}}\right|<\epsilon$.

(c) If $x=2$, then either $y_{n}=y_{0}+n \sqrt{\kappa-2}$ for all $n$, or $y_{n}=y_{0}-n \sqrt{\kappa-2}$ for all $n$. In particular, since we assume that $\kappa \neq 2,\left|y_{n}\right|$ grows linearly in $|n|$.

(d) If $x=-2$, then either $y_{n}=(-1)^{n}\left(y_{0}+n \sqrt{\kappa-2}\right)$ for all $n$, or $y_{n}=$ $(-1)^{n}\left(y_{0}-n \sqrt{\kappa-2}\right)$ for all $n$.

(e) If $x= \pm \sqrt{\kappa+2}$, then either $y_{n}=\lambda^{n} y_{0}$ for all $n$, or $y_{n}=\lambda^{-n} y_{0}$ for all $n$. In particular, if $x \notin[-2,2]$ and $y_{0} \neq 0$, then as $n \rightarrow \infty,\left|y_{n}\right| \rightarrow \infty$ (or 0$)$ and as $n \rightarrow-\infty,\left|y_{n}\right| \rightarrow 0($ resp. $\infty)$.

\section{Proofs of Theorems 1.2 and 1.3}

Fix $\rho \in \mathcal{X}_{\kappa}$, and again assume that $\kappa \neq 2$. For a pair of elements $X, Y \in \mathscr{C}$, recall that $\rho$ satisfies the extended BQ-conditions on $\mathscr{C}_{[X, Y]}$ if conditions (i) and (ii) of Theorem 1.3 are satisfied for all $Z \in \mathscr{C}_{[X, Y]}$ (2.13). It was shown in 10 that if this holds, then a version of the McShane's identity holds, in particular, a certain series converges which implies that for all $K>0$, the set $\left\{Z \in \mathscr{C}_{[X, Y]}|| z \mid \leq K\right\}$ is finite. Hence, we have

Proposition 4.1. Suppose that $\rho$ satisfies the extended BQ-conditions on $\mathscr{C}_{[X, Y]}$, then $\mathcal{E}(\rho) \cap \mathscr{C}_{(X, Y)}=\emptyset$. 
We shall see later (Proposition 4.3) that if in addition, $x, y \neq \pm \sqrt{\kappa+2}$, then in fact $\mathcal{E}(\rho) \cap \mathscr{C}_{[X, Y]}=\emptyset$.

Now suppose that $f_{\rho}(e)=\vec{e}$, with $\vec{e} \leftrightarrow\left(X, Y ; Z \rightarrow Z^{\prime}\right)$ and $X, Y \notin \mathscr{C}(2)$, that is, $|x|,|y|>2$. Then it was shown in [1] (see also 11]) that for all edges $e^{\prime} \in E(\Sigma)$ lying in the component of $\Sigma \backslash\{\vec{e}\}$ at the tail of $\vec{e}, f_{\rho}\left(e^{\prime}\right)$ points towards $\vec{e}$. In particular, for all $Z \in \mathscr{C}_{[X, Y]},|z| \geq \min (|x|,|y|)$ and hence tail $(\vec{e})=\mathscr{C}_{[X, Y]}$ satisfies the extended BQ-conditions. Therefore we have

Proposition 4.2. Suppose that $f_{\rho}(e)=\vec{e}$, with $\vec{e} \leftrightarrow\left(X, Y ; Z \rightarrow Z^{\prime}\right)$ and $X, Y \notin$ $\mathscr{C}(2)$. Then $\mathcal{E}(\rho) \cap \mathscr{C}_{(X, Y)}=\emptyset$.

Using the above result and Lemma 3.5 it is easy to determine which elements of $\mathscr{C}$ are in $\mathcal{E}(\rho)$, and also to show that $\mathcal{E}(\rho)$ is closed in $\mathscr{P} \mathscr{L}$.

Proposition 4.3. Suppose $X \in \mathscr{C}$. Then $X \in \mathcal{E}(\rho)$ if and only if $x \in(-2,2) \cup$ $\{ \pm \sqrt{\kappa+2}\}$.

Proof. It is clear from parts (b) and (e) of Lemma 3.5 that $X \in \mathcal{E}(\rho)$ if $x \in$ $(-2,2) \cup\{ \pm \sqrt{\kappa+2}\}$. Now suppose that $x \notin(-2,2) \cup\{ \pm \sqrt{\kappa+2}\}$. Then by parts (a), (c) and (d) of Lemma 3.5 for all $K>0$, there exists $N \in \mathbb{N}$ such that $\left|y_{n}\right|>K$ for all $n>N$ and $n<-N$. Hence, for all $K>0$, by the remark preceding Proposition 4.2 there exists a neighborhood $N(X)$ of $X$ (depending on $K$ ) such that $|z|>K$ for all $Z \in N(X) \backslash\{X\}$. We conclude that $X \notin \mathcal{E}(\rho)$.

Proposition 4.4. For any character $\rho \in \mathcal{X}$, the set of end invariants $\mathcal{E}(\rho)$ is closed in $\mathscr{P} \mathscr{L}$.

Proof. We show that $\mathscr{P} \mathscr{L} \backslash \mathcal{E}(\rho)$ is open. Suppose first that $X \in \mathscr{C}$ is not an end invariant. Then by Proposition 4.3] and Lemma 3.5 if $Y_{n}$ are the neighbors of $X,\left|y_{n}\right| \rightarrow \infty$ as $n \rightarrow \pm \infty$. Hence, by Propositions 4.2 4.3 there exists an open neighborhood $U$ of $X$ such that $U \cap \mathcal{E}(\rho)=\emptyset$. Now suppose $\lambda \in \mathscr{P} \mathscr{L} \backslash \mathscr{C}$ is not an end invariant. Let $\left\{e_{n}\right\}$ be a path in $\Sigma$ limiting to $\lambda$ and let $\left(X_{n}, Y_{n}\right) \in \mathcal{G P}$ be the generating pair corresponding to the edge $e_{n}$, oriented so that $\lambda \in\left[X_{n}, Y_{n}\right]$. Since $\lambda \notin \mathcal{E}(\rho)$, we have $\left|x_{n}\right|,\left|y_{n}\right| \rightarrow \infty$; so we may assume say that $\left|x_{1}\right|=K>2$, and both $\left|x_{N}\right|$ and $\left|y_{N}\right|>K$ for some $N \in \mathbb{N}$. By Lemma 3.1 $|z|>K$ for all $Z \in\left[X_{N}, Y_{N}\right]$, so that $\mathscr{C}_{\left[X_{N}, Y_{N}\right]}$ satisfies the extended BQ-conditions. Hence, $\left(X_{N}, Y_{N}\right) \cap \mathcal{E}(\rho)=\emptyset$ by Proposition 4.1. We conclude that $\mathscr{P} \mathscr{L} \backslash \mathcal{E}(\rho)$ is open.

Proof of Theorem 1.2 If $\rho$ is dihedral, then $x \in\{0, \pm \sqrt{\kappa+2}\}$ for all $X \in \mathscr{C}$, and if $\rho$ is a $\mathrm{SU}(2)$ character, then $x \in[-2,2]$ for all $X \in \mathscr{C}$. In either case, it is clear that $\mathcal{E}(\rho)=\mathscr{P} \mathscr{L}$ since $|x|$ is bounded for all $X \in \mathscr{C}$. Now suppose that $\mathcal{E}(\rho)$ contains an open interval $\mathcal{I} \subset \mathscr{P} \mathscr{L}$. Then there exists a generating triple $(X, Y, Z) \in \mathcal{G} \mathcal{T}$ with $X, Y, Z \in \mathcal{I}$. By Proposition $4.3 x, y, z \in(-2,2) \cup\{ \pm \sqrt{\kappa+2}\}$. Suppose say that $x= \pm \sqrt{\kappa+2}$, where $\sqrt{\kappa+2} \notin[-2,2]$. Then either $y=z=0$ in which case $\rho$ is a dihedral character, or $y, z \neq 0$, in which case by part (e) of Lemma 3.5 and Proposition 4.3 there exists a neighbor $Y_{n}$ of $X$ such that $Y_{n} \in \mathcal{I}$ but $Y_{n} \notin \mathcal{E}(\rho)$, which gives a contradiction. Hence, we may suppose that $x, y, z \neq \pm \sqrt{\kappa+2}$, in which case $x, y, z \in(-2,2)$ (recall $\kappa \neq 2$ ). If $\rho$ is a $\mathrm{SU}(2)$ character, $\mathcal{E}(\rho)=\mathscr{P} \mathscr{L}$ and we are done; otherwise, $\rho$ is a $\operatorname{SL}(2, \mathbb{R})$ character and there exists $W \in \mathscr{C}$ with $|w|>2, w \neq \pm \sqrt{\kappa+2}$ (see for example [3]) and so $W \notin \mathcal{E}(\rho)$. Since $x \in(-2,2)$, 
by Lemma 3.5(b), the neighboring values around $X$ are either periodic or quasiperiodic; it follows that there exists $W_{n}$ arbitrarily close to $X$ (hence in $\mathcal{I}$ ) with $W_{n} \notin \mathcal{E}(\rho)$. The contradiction completes the proof.

Proof of Theorem 1.3 Suppose that $\rho$ satisfies the extended BQ-conditions. It was shown in 10 that in this case, there exists a finite subtree of $\Sigma$ which is an attractor for the flow $f_{\rho}$ associated to $\rho$, and that the generalized McShane's identity holds. This implies that for any $K>0$, the set $\{X \in \mathscr{C}|| x \mid \leq K\}$ is finite. It follows that $\mathcal{E}(\rho)=\emptyset$. Note that Lemmas 3.1 and 3.4 played essential roles in the proof, in particular, if the extended BQ-conditions are satisfied, there cannot be an escaping orbit in the sense of Lemma 3.4 which is a crucial step towards showing the existence of the attractor.

Conversely, if $\rho$ does not satisfy the extended BQ-conditions, then either there exists some $X \in \mathscr{C}$ with $x \in(-2,2)$ or the set $\mathscr{C}(2)=\{X \in \mathscr{C}|| x \mid \leq 2\}$ is infinite. In the first case, $X \in \mathcal{E}(\rho)$, and in the second case, $\mathscr{C}(2)$ has an accumulation point in $\mathscr{P} \mathscr{L}$ which lies in $\mathcal{E}(\rho)$.

\section{Reducible Characters}

We consider the reducible characters in this section and prove Theorem 1.4 As pointed out in 2.16 we may use representations into diagonal matrices of $\mathrm{SL}(2, \mathbb{C})$ to represent the reducible characters. Note that $\rho\left(X Y X^{-1} Y^{-1}\right)=I$ for all generating pairs $X, Y$ of $\pi$, where $I$ is the identity matrix. Hence we may think of $\rho$ as corresponding to a representation of the fundamental group of the torus $\mathbb{T}$ (without boundary) and use the homology classes $\left[X^{m} Y^{n}\right]$ where $m, n$ are relatively prime and $n \geq 0$ to represent the elements of $\mathscr{C}$. Let $\pi(\mathbb{T})=\langle X, Y| X Y X^{-1} Y^{-1}=$ $I\rangle$, then

$$
\rho(X)=\left(\begin{array}{cc}
\alpha^{1 / 2} & 0 \\
0 & \alpha^{-1 / 2}
\end{array}\right), \quad \rho(Y)=\left(\begin{array}{cc}
\beta^{1 / 2} & 0 \\
0 & \beta^{-1 / 2}
\end{array}\right),
$$

where $\alpha, \beta \in \mathbb{C}$, and $x=\operatorname{tr} \rho(X)=\alpha^{1 / 2}+\alpha^{-1 / 2}, y=\operatorname{tr} \rho(Y)=\beta^{1 / 2}+\beta^{-1 / 2}$. There are two cases to consider, (i) $p \cdot \log |\alpha|+q \cdot \log |\beta|=0$ for some integers $p, q$ (not all zero); and (ii) otherwise. In the first case, we have $\left|\alpha^{p} \cdot \beta^{q}\right|=1$. By using the action of $\Gamma$, we might as well assume that in fact $p=1$ and $q=0$, so that $|\alpha|=1$, and $x \in[-2,2]$. If $|\beta|=1$ as well, then $y \in[-2,2]$ and similarly $z=\operatorname{tr} \rho(X Y) \in[-2,2]$. Since $\kappa=2, \rho$ corresponds to a $\mathrm{SU}(2)$ representation and $\mathcal{E}(\rho)=\mathscr{P} \mathscr{L}$ in this case. If $|\beta| \neq 1$, then $|z| \notin[-2,2]$ for all other $Z \in \mathscr{C} \backslash\{X\}$, since the exponent of $Y$ is non-zero for all the other elements. Furthermore, since $\left|\alpha^{n} \beta\right|=|\beta|$ is bounded, $X \in \mathcal{E}(\rho)$. For any other element $Z \in \mathscr{P} \mathscr{L}$, if $Z_{n}$ is any sequence of distinct elements approaching $Z$, then the exponent of $Y$ in the homology class of $Z_{n}$ approaches $\pm \infty$, so that $\log \left|Z_{n}\right| \rightarrow \pm \infty$. Hence $Z \notin \mathcal{E}(\rho)$. It follows that in this case, $\mathcal{E}(\rho)=\{X\}$. Now we consider case (ii), where $\frac{\log |\alpha|}{\log |\beta|}=\mu$ is an irrational number. There exists a sequence of rationals $p_{n} / q_{n} \rightarrow \mu$, namely, the convergents of $\mu$ in the continued fraction expansion of $\mu$ such that $p_{n} \cdot \log |\alpha|+q_{n} \cdot \log |\beta|$ is bounded. Hence $X(\mu) \in \mathscr{P} \mathscr{L}$ lies in $\mathcal{E}(\rho)$. For any other $Z \in \mathscr{P} \mathscr{L}$, if $X\left(p_{n} / q_{n}\right)$ is any sequence of elements approaching $Z$, then $p_{n} \cdot \log |\alpha|+q_{n} \cdot \log |\beta|$ is unbounded and hence $Z \notin \mathcal{E}(\rho)$. It follows that $\mathcal{E}(\rho)=\{X(\mu)\}$ in this case. This completes the proof of Theorem 1.4 


\section{Proofs of Theorems 1.5 and 1.7}

We first give an equivalent definition for the set $\mathcal{E}(\rho)$ in terms of the (projectivized) ends of a certain subtree $\mathcal{H}$ of $\Sigma$; this is the variation of the original definition given by Bowditch in 1 .

Suppose $\rho \in \mathcal{X}$ does not satisfy the extended BQ-conditions. We define a subtree $\mathcal{H}:=\mathcal{H}_{\rho}$ of $\Sigma$ as follows: Suppose that $e \in E(\Sigma)$ corresponds to the generating pair $(X, Y)$. Then $e \subset \mathcal{H}$ if both $\mathscr{C}_{[X, Y]}$ and $\mathscr{C}_{[Y, X]}$ does not satisfy the extended BQ-conditions.

For edges $e \in E(\Sigma) \backslash \mathcal{H}$, exactly one of the sets $\mathscr{C}_{[X, Y]}, \mathscr{C}_{[Y, X]}$ does not satisfy the extended BQ-conditions (since $\rho$ doesn't); we define a direction $d(e) \in \vec{E}(\Sigma)$ so that tail of $d(e)$ satisfies the extended BQ-conditions. Hence, $d(e)$ points towards $\mathcal{H}$ for all $e \notin \mathcal{H}$. Note that $d(e)$ is not to be confused with the flow $f(e)$ defined in 2.18 It is easy to see from the definition that any vertex $v \in V(\Sigma)$ has 0,2 or 3 edges of $\mathcal{H}$ adjacent to it. A vertex $v \in \mathcal{H}$ is called a node of $\mathcal{H}$ if there are three edges of $\mathcal{H}$ adjacent to it. Furthermore, if $v$ is not a vertex of $\mathcal{H}$, then for the three edges incident at $v, d(e)$ points towards $v$ for two of the edges and away from $v$ for the third. If $v$ has 2 edges of $\mathcal{H}$ adjacent to it, then $d(e)$ points towards $v$ for the remaining edge.

There is a natural projection map from the ends of $\Sigma$ to $\mathscr{P} \mathscr{L}$ which is one to one onto irrational points and two to one to rational points. If $\mathcal{H}$ is empty then $d(e)$ all point towards a unique $\lambda \in \mathscr{P} \mathscr{L}$ and $\mathcal{E}(\rho)=\{\lambda\}$; otherwise, $\mathcal{E}(\rho)$ is the image of the ends of $\mathcal{H}$ under the projection map to $\mathscr{P} \mathscr{L}$.

For an irrational $\lambda \in \mathcal{E}(\rho)$, the following result states that we can take $K=2$ in the definition of $\mathcal{E}(\rho)$.

Proposition 6.1. Suppose that $\lambda \in \mathcal{E}(\rho)$ and $\lambda \notin \mathscr{C}$. Then there exists a sequence of distinct elements $Z_{n} \in \mathscr{C}$ such that $Z_{n} \rightarrow \lambda$ and $\left|z_{n}\right| \leq 2$ for all $n$.

Proof. If $\mathcal{H}$ is empty, then $d(e)$ points towards $\lambda$ for all $e \in E(\Sigma)$. Choose any path $\left\{e_{n}\right\}$ limiting at $\lambda$ and let $\left(X_{n}, Y_{n}\right)$ be the generating pair corresponding to $e_{n}$, ordered so that $\lambda \in\left[X_{n}, Y_{n}\right]$. Then $\rho$ does not satisfy the extended BQconditions on $\mathscr{C}_{\left[X_{n}, Y_{n}\right]}$, and hence, there exists $Z_{n} \in \mathscr{C}_{\left[X_{n}, Y_{n}\right]}$ such that $\left|z_{n}\right| \leq 2$. Since $X_{n}, Y_{n} \rightarrow \lambda$, by passing to a subsequence if necessary, we obtain a sequence of distinct $Z_{n} \rightarrow \lambda$ with $\left|z_{n}\right| \leq 2$. The same argument works if $\lambda$ is the end of a path in $\mathcal{H}$ : we just choose a path in $\mathcal{H}$ ending at $\lambda$.

Proof of Theorem 1.5 Let $\rho \in \mathcal{X}_{\kappa}^{\mathbb{R}}$ with $\phi$ the corresponding Fricke trace map, and suppose that $\kappa \neq 2$. We first prove the following claim:

Claim. If $|\mathcal{E}(\rho)|>1$, then $|\mathcal{E}(\rho) \cap \mathscr{C}|=\infty$, and if $\mathcal{E}(\rho)=\{X\}$, then $X \in \mathscr{C}$. Furthermore, if $|\mathcal{E}(\rho)|>1$, then $\mathcal{E}(\rho)$ is perfect (that is, every element of $\mathcal{E}(\rho)$ is an accumulation point of $\mathcal{E}(\rho))$.

First note that $x=\phi(X) \in \mathbb{R}$ for all $X \in \mathscr{C}$ by the edge relation (8). Hence by Proposition 4.3 $X \in \mathcal{E}(\rho)$ if $|x|<2$. If there exists two distinct $X, Y \in \mathscr{C}$ with $|x|,|y|<2$, then by looking at the behavior of $\phi$ about $X$ and $Y$ respectively and applying Lemma 3.5 (b), we see that there are infinitely many $Z \in \mathscr{C} \cap \mathcal{E}(\rho)$ and both $X$ and $Y$ are accumulation points in $\mathcal{E}(\rho)$. Similarly, if there exists $X \in \mathscr{C} \cap \mathcal{E}(\rho)$ with $x= \pm \sqrt{\kappa+2}$, and $\sqrt{\kappa+2}>2$, then by Lemma 3.5 (e), there are infinitely many $Z \in \mathscr{C} \cap \mathcal{E}(\rho)$ and $X$ is an accumulation point of $\mathcal{E}(\rho)$ (in fact, it is a one 
sided limit point of $\mathcal{E}(\rho)$ if $\rho$ is not dihedral). Now suppose that $\lambda \in \mathcal{E}(\rho)$ with $\lambda \notin \mathscr{C}$. By Proposition 6.1] $|\mathscr{C}(2)|=\infty$ and since $\mathscr{C}(2)$ is connected, we can find a sequence $\left\{X_{n}\right\} \subset \mathscr{C}(2)$ such that $X_{n} \rightarrow \lambda$ and $\left(X_{n}, X_{n+1}\right)$ is a generating pair for all $n$. If there are infinitely many $X_{k}$ in the sequence such that $\left|x_{k}\right|<2$, we are done. Otherwise, re-indexing if necessary, we may suppose that $x_{n}= \pm 2$ for all $n \in \mathbb{N}$. Note that $X_{k-1}$ and $X_{k+1}$ are neighbors of $X_{k}$. If $\left(X_{k-1}, X_{k}, X_{k+1}\right)$ is a generating triple, then using the sign-change automorphism (2.4), we may assume that $\left(x_{k-1}, x_{k}, x_{k+1}\right)=(2,2,2)$ or $(-2,-2,-2)$. In the first case, $\kappa=2$ which contradicts our assumption. The second case corresponds to the holonomy representation of the thrice punctured sphere and $\phi$ satisfies the extended BQconditions, so $\mathcal{E}(\rho)=\emptyset$, again a contradiction. Hence, $\left(X_{k-1}, X_{k}, X_{k+1}\right)$ is not a generating triple and there exists $Z_{k}$, a neighbor of $X_{k}$ lying between $X_{k-1}$ and $X_{k+1}$. Again, by the sign change automorphism, we may assume that $x_{k}=2$. Now by Lemma 3.5 (c), $x_{k-1}$ and $x_{k+1}$ have opposite signs, say $x_{k-1}=-2$ and $x_{k+1}=2$, which forces $\left|z_{k}\right|<2$. This produces a sequence of elements $Z_{k} \in \mathcal{E}(\rho) \cap \mathscr{C}$ approaching $\lambda$ which completes the proof of the claim.

The classification of the types for $\mathcal{E}(\rho)$ according to (a), (b), (c) or (d) of the theorem now follows from the above claim and Theorems 1.2 and 1.3 It remains to show that each of these cases occur for the values of $\kappa$ stated. We use here Goldman's main Theorem in 3] which classifies the action of $\Gamma$ on $\mathcal{X}_{\kappa}^{\mathbb{R}}$. The fact that case (a) occurs if and only if $\kappa<2$ or $\geq 18$ follows from the fact that $\Gamma$ acts properly only on characters in these ranges of $\kappa$. The ranges for case (c) and (d) also follow from Goldman's result, Theorem 1.2 the above argument, and the fact that the dihedral character is real for $\kappa>-2$. It remains to show that case (b) occurs if and only if $\kappa \geq 6$. It is easy to see that $\mathcal{E}(\rho)=\{X\}$ if $\iota(\rho)=(0, a, a)$, where $a \geq 2$. Since in this case $\kappa=2 a^{2}-2 \geq 6$, we have case (b) occurs for all $\kappa \geq 6$. Conversely, we show that if case (b) occurs, that is, if $\mathcal{E}(\rho)=\{X\}$, then $\kappa \geq 6$. Let $Y_{n}$, where $n \in \mathbb{Z}$, be the neighbors of $X$. Using the sign change automorphism, we may assume that $x \geq 0$, so that $x \in[0,2), x=e^{i \theta}+e^{-i \theta}$ where $0<\theta \leq \pi / 2$, and there exist $A, B \in \mathbb{C}$ with $A B=\left(x^{2}-\kappa-2\right) /\left(x^{2}-4\right)$ such that $y_{n}=A e^{i n \theta}+B e^{-i n \theta}$. Since $y_{n} \in \mathbb{R}$ for all $n$, we have $B=\bar{A}$ so that $y_{n}=2 \operatorname{Re}\left(A e^{i n \theta}\right)$. Now since $\left|y_{n}\right| \geq 2$ for all $n, \theta$ must be a rational multiple of $\pi$, that is, $\theta=p \pi / q$ for some rational $p / q$, and the set $\left\{A e^{i n \theta}\right\}$ is the set of vertices of a regular $q$-gon in the complex plane centered at the origin with $\left|2 \operatorname{Re}\left(A e^{i n \theta}\right)\right| \geq 2$ for all $n$. Re-indexing if necessary, we may assume that $y_{0}=2 \operatorname{Re}(A)>0$; hence $y_{0} \geq 2$, and $y_{1}=2 \operatorname{Re}\left(A e^{i \theta}\right)<0$, which implies $y_{1} \leq-2$. It follows that $\kappa=x^{2}+y_{0}^{2}+y_{1}^{2}-x y_{0} y_{1}-2 \geq 8-2=6$ which completes the proof of Theorem 1.5

Proof of Theorem [1.7 Suppose that $\rho$ is discrete, and $\mathcal{E}(\rho)$ has at least three elements but is not equal to $\mathscr{P} \mathscr{L}$. By Theorem 1.2 it suffices to prove that $\mathcal{E}(\rho)$ is perfect, that is every $X \in \mathcal{E}(\rho)$ is the limit of distinct $Z_{n} \in \mathcal{E}(\rho)$. Note that in this case $\mathcal{H}$ has at least three distinct ends and at least one node.

Case 1. $X \in \mathscr{C} \cap \mathcal{E}(\rho)$. By Proposition 4.3 and the discreteness of $\rho, x=$ $2 \cos \frac{p}{q} \pi$ for some $\frac{p}{q} \in \mathbb{Q}$, and by the periodicity of $\phi$ about $X$ ( $\rho$ is stabilized by a reducible element of the mapping class group $\Gamma$ fixing $X$ ) and the fact that $\mathcal{H}$ has at least 3 ends, $X$ is a limit of distinct $Z_{n} \in \mathcal{E}(\rho)$.

Case 2: $X \notin \mathscr{C}$. Choose a node $v$ of $\mathcal{H}$ (which exists by the assumption) and the path $\left\{e_{n}\right\}$ from $v$ to $X$ and let $\left(X_{n}, Y_{n}\right)$ be the generating pair corresponding to 
$e_{n}$, ordered so that $X \in\left[X_{n}, Y_{n}\right]$. By the connectedness of $\mathscr{C}(2)$ (Lemma 3.1) and the vertex relation (17), it is easy to see that there exists a universal constant $K>0$ depending only on $\kappa$ such that $\left|x_{n}\right|,\left|y_{n}\right|<K$. Since the set of values are discrete, by passing to a subsequence, we obtain a sequence of generating pairs $\left(X_{n}, Y_{n}\right)$ approaching $X$ such that for all $n \in \mathbb{N}, x_{n}=a, y_{n}=b$ where $a, b \in \mathbb{C}$ are fixed constants. Let $d$ be the distance from $v$ to the edge $e_{1} \leftrightarrow\left(X_{1}, Y_{1}\right)$. It follows that there is a node $v_{n}$ of $\mathcal{H}$ at distance $d$ from each of $e_{n} \leftrightarrow\left(X_{n}, Y_{n}\right)$. Since $e_{n} \rightarrow X$, $v_{n} \rightarrow X$, and hence $X$ is the limit point of distinct $\lambda_{n} \in \mathcal{E}(\rho)$.

Remark 6.2. We end this section by remarking that if $\rho \in \mathcal{X}^{\text {disc }}$ satisfies the conditions of Theorem 1.7 and $\mathcal{E}(\rho) \neq \mathscr{P} \mathscr{L}$, then the proof of Theorem 1.7 implies that in fact, the stabilizer of $\rho$ in the mapping class group $\Gamma$ of $T$ is relatively large, in particular, is not finite or virtually cyclic. This answers a question posed by Bowditch in [1. Makoto Sakuma 9] has also independently obtained examples of characters whose stabilizer in $\Gamma$ is not finite or virtually cyclic by considering the representations arising from the two bridge knot complements; in these cases there are at least two (hence infinitely many) $X \in \mathscr{C}$ for which $\phi(X)=0$.

\section{Proof of Theorem 1.6}

For the rest of this section, we fix $\rho \in \mathcal{X}_{\kappa}^{I}$, where $\kappa<2$, with corresponding Fricke trace map $\phi$. It will be convenient and visually easier to regard $\phi$ as a map from $\Omega(\Sigma)$ to $\mathbb{C}$. Recall from 2.10 that there is a partition of $\mathscr{C}$ and $\Omega(\Sigma)$ into three equivalence classes $\mathcal{R}, \mathcal{G}$ and $\mathcal{B}$. We use the letters $Z, Y$ and $X$ to denote the elements of $\mathcal{R}, \mathcal{G}$ and $\mathcal{B}$ respectively, and $W$ to denote a general element of $\mathscr{C}$. Since $\rho$ is imaginary, $\phi$ takes purely imaginary values on two of the equivalence classes and real values on the third; we may assume that it takes real values on $Z \in \mathcal{R}$. For $X \in \mathcal{B}, Y \in \mathcal{G}$ and $Z \in \mathcal{R}$, we use the convention $\phi(X)=i x$, $\phi(Y)=i y$ and $\phi(Z)=z$, where $x, y, z \in \mathbb{R}$ for the rest of this section (note that this is different from the convention used earlier). Then if $(X, Y, Z) \in \mathcal{G} \mathcal{T}$ and $\left(X, Y ; Z, Z^{\prime}\right),\left(Y, Z ; X, X^{\prime}\right),\left(Z, X ; Y, Y^{\prime}\right) \in \mathcal{G} \mathcal{Q}$, the vertex relation (77) and edge relation (8]) can be rewritten as:

$$
\begin{gathered}
-x^{2}-y^{2}+z^{2}+x y z-2=\kappa \\
z+z^{\prime}=-x y, \quad y+y^{\prime}=x z, \quad x+x^{\prime}=y z
\end{gathered}
$$

Note that by our convention (2.15) $\rho \in \mathcal{X}_{\kappa}^{I}$ is not dihedral. Hence, by Theorem $1.2 \mathcal{E}(\rho) \neq \mathscr{P} \mathscr{L}$, and in fact $\mathcal{E}(\rho)$ has empty interior in $\mathscr{P} \mathscr{L}$. We will be using the results of Goldman-Stantchev, in particular, the classification result (Theorem A of [5]) and the $\tau$-reduction algorithm ( $\S 4$ of [5]). The following strengthening of Proposition 6.1 concerning irrational ends is key.

Proposition 7.1. Suppose $\rho \in \mathcal{X}_{\kappa}^{I}$, where $\kappa<2$ and $\lambda \in \mathcal{E}(\rho)$ with $\lambda \notin \mathscr{C}$. Then there exists a sequence of distinct elements $\left\{W_{n}\right\} \subset \mathscr{C} \cap \mathcal{E}(\rho)$ such that $W_{n} \rightarrow \lambda$.

Proof. By Proposition 6.1 $\Omega(2) \neq \emptyset$. Choose $W_{0} \in \Omega(2)$ and let $P=\left\{e_{n}\right\}$ be the minimal path in $\Sigma(\Omega)$ from $W_{0}$ limiting at $\lambda$. By Lemma 3.1 we can find a sequence $\left\{W_{n}\right\} \subset \Omega(2)$ such that for all $n \geq 1, W_{n}$ is adjacent to $P, W_{n}, W_{n+1}$ are neighbors and $W_{n} \rightarrow \lambda$ (see Figure 3). We consider two cases, first when $W_{n} \in \mathcal{R}$ for infinitely many $n$, and secondly, when $W_{n} \notin \mathcal{R}$ for all but finitely many $n$. 


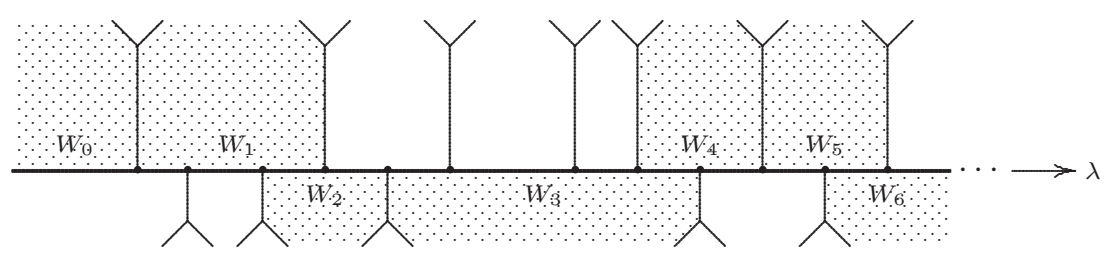

Figure 3 . The sequence $\left\{W_{n}\right\} \longrightarrow \lambda$ along $P$.

Case 1. $W_{n} \in \mathcal{R}$ for infinitely many $n$. This gives a subsequence $\left\{Z_{n}\right\} \subset \mathcal{R}$ approaching $\lambda$ such that $\left|z_{n}\right| \leq 2$. If there are infinitely many elements in this sequence with $\left|z_{n}\right|<2$, these are in $\mathcal{E}(\rho)$ by Proposition 4.3 so we are done. Hence we might as well assume that $z_{n}= \pm 2$ for all $n$. Then there exists infinitely many $k$ such that $\phi\left(W_{k}\right)= \pm 2$ in the sequence $\left\{W_{n}\right\}$. Fix such a $k$ and consider the triple $W_{k-1}, W_{k}, W_{k+1}$. Using the sign-change automorphism (2.4) we might as well assume that $w_{k}=2 . W_{k-1}$ and $W_{k+1}$ are neighbors of $W_{k}$ (hence, not in $R$ ), with $\phi\left(W_{k-1}\right)=i w_{k-1}, \phi\left(W_{k+1}\right)=i w_{k+1}$, where $w_{k-1}, w_{k+1} \in[-2,2]$, by assumption. If $w_{k-1}=0\left(\right.$ or $\left.w_{k+1}=0\right)$, then $W_{k-1} \in \mathcal{E}(\rho)\left(\operatorname{resp} . W_{k+1} \in \mathcal{E}(\rho)\right)$. If both $w_{k-1}$ and $w_{k+1}$ have the same sign, then, since the values of the neighbors of $W_{k}$ grow linearly (Lemma 3.5(c)), the difference in the values of successive neighbors of $W_{k}$ is $c i$, where $0<c<2$, and we can find two successive neighbors, say $X$ and $Y$, of $W_{k}$ such that $\phi(X)=i x, \phi(Y)=i y$ with $-2 \leq x<0<y \leq 2$. If $w_{k-1}$ and $w_{k+1}$ have different signs, then a simple argument using the neighbors of $W_{k}$ in between $W_{k-1}$ and $W_{k+1}$ again yields neighbors $X, Y$ of $W_{k}$ with the same property. If $x=-2$ and $y=2$, we have $\left(w_{k}, i x, i y\right)=(2,-2 i, 2 i)$, and $\rho$ can be easily shown to satisfy the extended BQ-conditions (see [11), so that $\mathcal{E}(\rho)=\emptyset$, contradicting the fact that $\rho$ has an irrational end. Hence, we may assume that either $x>-2$ or $y<2$. Now if we consider the generating quadruple $\left(X, Y ; W_{k}, Z_{k}\right)$, we have $\left|z_{k}\right|=|-x y-2|<2$, so we obtain $Z_{k} \in \mathcal{R} \cap \mathcal{E}(\rho)$. In all subcases, we obtain a subsequence $\left\{W_{n}\right\} \subset \mathscr{C} \cap \mathcal{E}(\rho)$ such that $W_{n} \rightarrow \lambda$, which completes the proof in this case.

Case 2. $W_{n} \notin \mathcal{R}$ for all but finitely many $n$. Re-indexing if necessary, we might as well assume that $W_{n} \notin \mathcal{R}$ for all $n$, and $\left\{W_{n}\right\}=X_{1}, Y_{1}, X_{2}, Y_{2}, \ldots$, where $X_{n} \in \mathcal{B}, Y_{n} \in \mathcal{G}$. The sequence $\left\{W_{n}\right\}$ must cross the path $P$ infinitely often, since the end of $P$ is irrational, and $W_{n} \notin \mathcal{R}$ for all $n$. Hence, renaming $\mathcal{G}$ and $\mathcal{B}$ if necessary, we obtain a subsequence of generating pairs $\left\{\left(X_{n}, Y_{n}\right)\right\} \subset \mathcal{G P}$ and a nested sequence of closed intervals $\left[X_{n}, Y_{n}\right]$ such that

$$
\lambda \in \cdots \subset\left[X_{n+1}, Y_{n+1}\right] \subset\left[X_{n}, Y_{n}\right] \cdots \subset\left[X_{1}, Y_{1}\right],
$$

$X_{n}, Y_{n} \rightarrow \lambda$, and $\left|i x_{n}\right|,\left|i y_{n}\right| \leq 2$ for all $n \geq 1$. Passing again to a subsequence, we may assume that $x_{n} \rightarrow a, y_{n} \rightarrow b$, where $a, b \in[-2,2]$. Let $e_{n} \in E_{r} \subset E(\Sigma)$ be the edge of $P$ corresponding to the generating pair $\left(X_{n}, Y_{n}\right)$ and $v_{n} \in V(\Sigma)$ be the end of $e_{n}$ which is closer to $\lambda$. Note that the quantity $\tau_{n}=-z_{n} z_{n}^{\prime}$ associated to the edge $e_{n} \leftrightarrow\left(X_{n}, Y_{n} ; Z_{n}, Z_{n}^{\prime}\right) \in \mathcal{G} \mathcal{Q}$ approaches a fixed constant $c$ which depends only on $a$ and $b$. We now consider the case where both $a, b \neq 0$ and the case where one of $a, b=0$ separately, and perform the $\tau$-reduction algorithm at each $v_{n}$. 
Subcase (i): $a, b \neq 0$. Then, starting at $v_{n}$, the $\tau$-reduction algorithm of Goldman-Stantchev [5], see also the Appendix, produces a sequence in $\mathscr{C}$ terminating after a finite number of steps at some $Z_{n} \in \mathcal{E}(\rho)$. For $n$ sufficiently large, since $a, b \neq 0$, the first (two) steps of the algorithm reduces $\tau$ by at least some positive constant depending only on $a$ and $b$, hence if $n$ is sufficiently large, the algorithm starting at $v_{n}$ does not cross the edges $e_{n-1} \leftrightarrow\left(X_{n-1}, Y_{n-1}\right)$ and $e_{n+1} \leftrightarrow\left(X_{n+1}, Y_{n+1}\right)$. Hence, $Z_{n} \in\left[X_{n-1}, Y_{n-1}\right] \backslash\left[X_{n+1}, Y_{n+1}\right]$. Again passing to a subsequence, we obtain a sequence of distinct $Z_{n} \in \mathcal{E}(\rho)$ approaching $\lambda$.

Subcase (ii): One of $a, b=0$, say $a=0$. Then for any $\epsilon>0$, there exists $N$ such that $\left|i x_{n}\right|<\epsilon$ for all $n>N$. Again, the $\tau$-reduction algorithm starting at $v_{n}$ terminates, after a finite number of steps, at some $Z_{n} \in \mathscr{C}$ such that $Z_{n} \in \mathcal{E}(\rho)$. Furthermore, if $\left|i x_{n}\right|$ is sufficiently small, by [5] (see Appendix), $Z_{n}$ is in fact a neighbor of $X_{n}$. Again it follows that for $n$ sufficiently large, $Z_{n} \in\left[X_{n-1}, Y_{n-1}\right] \backslash$ $\left[X_{n+1}, Y_{n+1}\right]$, and the conclusion follows as in Subcase (i).

Proof of Theorem [1.6] The proof for Theorem [1.6] now follows easily, along similar lines to the proof of Theorem 1.5 If $|\mathcal{E}(\rho) \cap \mathscr{C}|>1$, it follows that $\mid \mathcal{E}(\rho) \cap$ $\mathscr{C} \mid=\infty$ by Lemma 3.5 (b), and furthermore, each $W \in \mathcal{E}(\rho) \cap \mathscr{C}$ is an accumulation point of $\mathcal{E}(\rho)$. If $\mathcal{E}(\rho)$ has an irrational end $\lambda$, it follows from Proposition 7.1 that $|\mathcal{E}(\rho) \cap \mathscr{C}|=\infty$ and $\lambda$ is an accumulation point of $\mathcal{E}(\rho)$. Hence, $\mathcal{E}(\rho)$ is either empty, has one element $W \in \mathscr{C}$ or is a Cantor set. The fact that $\mathcal{E}(\rho)=\emptyset$ occurs only for $\kappa \leq-14$ and not for $-14<\kappa<2$ follows from [5] (actually, the case $\kappa=-14$ was not covered, but as we saw earlier, the example $\rho \in \mathcal{X}_{-14}$ where $\iota(\rho)=(2,2 i,-2 i)$ has $\mathcal{E}(\rho)=\emptyset)$. It is fairly easy to construct, for all $\kappa<2$, examples where $\mathcal{E}(\rho)=\{X\}$ : we use $\rho$ with $\iota(\rho)=(0, y i, z)$ where $y, z$ are chosen such that $y, z>2$ and $z^{2}-y^{2}=\kappa+2$. In this case $\mathscr{C}(2)=\mathcal{E}(\rho)=\{X\}$. Similarly, it is easy to construct examples where $|\mathcal{E}(\rho)|>1$, and hence is a Cantor set: we just need to make sure that there are at least two element in $\mathscr{C} \cap \mathcal{E}(\rho)$. We leave this to the reader. Finally, to prove part (i) of the Theorem, we first show that for $\rho \in \mathcal{X}_{-2}$, if $\mathcal{E}(\rho)=\{W\}$, then $W \notin \mathcal{R}$. Suppose not, then we have some $\left(X_{0}, Y_{0}, Z_{0}\right) \in \mathcal{G} \mathcal{T}$ such that $z_{0} \in(-2,2)$. If $z_{0}=0$, then $i x_{0}=i y_{0}=0$, a contradiction since $\mathcal{E}(\rho)$ has only one element. Hence, using the sign change automorphism if necessary, we may assume that $z_{0} \in(0,2)$. Use $X_{n}, Y_{n}$ to denote the successive neighbors of $Z_{0}$, with values $i x_{n}, i y_{n}$ respectively. Then $x_{n}, y_{n}$ lie on the ellipse

$$
x^{2}-z_{0} x y+y^{2}=z_{0}^{2},
$$

with major axis $y=x$ and minor axis $y=-x$, and intercepts $\left( \pm z_{0}, 0\right)$ and $\left(0, \pm z_{0}\right)$. The values $x_{n}, y_{n}$ are obtained by starting at the point $\left(x_{0}, y_{0}\right)$ on the ellipse and taking the coordinates of the successive intersections of the ellipse with the up/down and left/right path; see Figure 4 (compare with [5]). There is at least one intercept which lies in either the second or the fourth quadrant, that is, there exist some successive neighbors $X_{n}, Y_{n}$ (or $Y_{n}, X_{n+1}$ ) of $Z_{0}$ such that $x_{n}, y_{n}$ have opposite signs and $\left|x_{n}\right|,\left|y_{n}\right| \leq z_{0}<2$. However, in this case, for the generating quadruple $\left(X_{n}, Y_{n} ; Z_{0}, Z_{n}\right) \in \mathcal{G} \mathcal{Q},\left|z_{n}\right|=\left|x_{n} y_{n}-z_{0}\right|<2$, contradicting the fact that $\mathcal{E}(\rho)$ has only one element. Hence we conclude that the single element $W$ of $\mathcal{E}(\rho)$ is not in $\mathcal{R}$; so it must be in $\mathcal{G}$ or $\mathcal{B}$, which implies $w=0$. The conclusion then follows easily from the vertex relation (7) and the connectedness of $\mathscr{C}(2)$. 


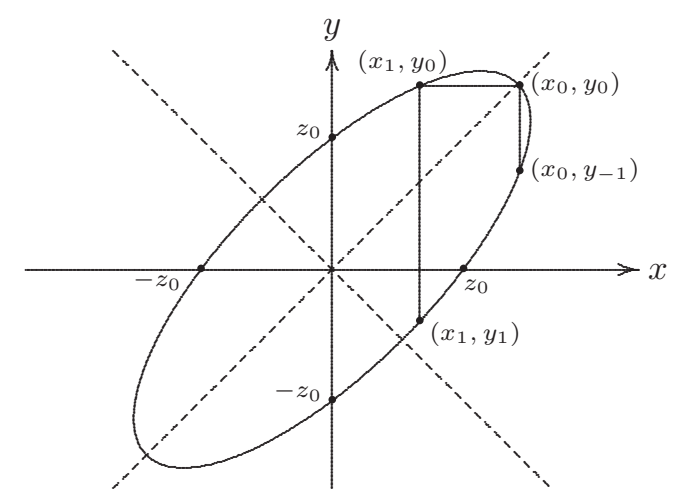

FiguRE 4 . The ellipse $x^{2}-z_{0} x y+y^{2}=z_{0}^{2}\left(z_{0}=1.5\right)$ and successive values of $x_{n}, y_{n}$.

\section{Appendix: The $\tau$-Reduction ALgorithm}

We give a brief description of the $\tau$-reduction algorithm given by Goldman and Stantchev in 5]. Bowditch's algorithm in 11 for type-preserving imaginary Markoff maps $\left(\rho \in \mathcal{X}_{-2}^{I}\right)$ is essentially a special case of this, although it was couched in a different setting using the Jorgenson parameters, $a=x / y z, b=y / x z$ and $c=z / x y$ instead of $x, y, z$. We will describe the results and algorithm but refer the reader to $[5$ for detailed proofs.

Fix a $\rho \in \mathcal{X}_{\kappa}^{I}$, where $\kappa<2$, with corresponding $\phi$. We adopt the notation in $\$ 7$ in particular, we use $X, Y$ and $Z$ for the elements of $\mathcal{B}, \mathcal{G}$ and $\mathcal{R}$ respectively (thought of as subsets of $\Omega(\Sigma)$ ), and write $z=\phi(Z)$, ix $=\phi(X)$, iy $=\phi(Y)$ where $x, y, z \in \mathbb{R}$. If $e \in E_{r} \subset E(\Sigma)$ corresponds to the generating quadruple $\left(X, Y ; Z, Z^{\prime}\right)$, define $\tau(e)=-z z^{\prime}$. Similarly, for $v \in V(\Sigma)$, define $\tau(v)=\tau(e)$ where $e$ is the unique edge in $E_{r}$ with one endpoint at $v$. Note that $\tau$ is invariant under the sign-change automorphism.

The $\tau$-reduction algorithm has as a starting point a vertex $v_{0} \in V(\Sigma)$, and produces a (unique) finite sequence of adjacent vertices $v_{0}, v_{1}, \cdots, v_{N}$ such that either

(a) $\tau\left(v_{N}\right)$ in minimal among all vertices $v \in V(\Sigma), v_{N}$ is an attractor for the flow $f_{\rho}, \phi$ satisfies the extended BQ-conditions and $\mathcal{E}(\rho)=\emptyset$; or

(b) one of the three regions adjacent to $v_{N}$ is in $\mathcal{E}(\rho)$.

This also produces a sequence of elements $W_{1}, W_{2}, \cdots, W_{N} \in \Omega(\Sigma)$, where $W_{k}$ is the (unique) region adjacent to $v_{k}$ which is not adjacent to $v_{k-1}$. The algorithm consists of two general types of moves, the first type moves the vertices around the boundary of $Z \in \mathcal{R}$ until we reach a point where $\tau$ is minimum among all the vertices lying on the boundary of $Z$, then the second type "flips" the vertex across the edge $e \in E_{r}$ to a vertex which is now adjacent to a different $Z^{\prime} \in \mathcal{R}$. The main point is that the algorithm is uniquely determined, does not backtrack, and 


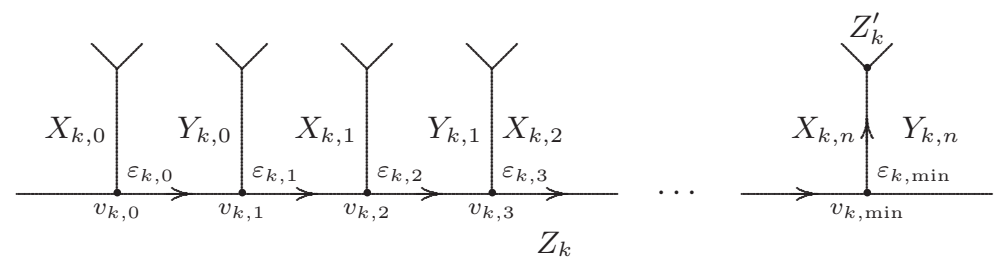

FiguRE 5. $\tau$-algorithm moves along $\partial Z_{k}$

terminates after a finite number of steps in one of the two possibilities (a) or (b) listed above.

The algorithm: Let $v_{0} \leftrightarrow\left(X_{0}, Y_{0}, Z_{0}\right) \in \mathcal{G T}$, and $e \leftrightarrow\left(X_{0}, Y_{0} ; Z_{0}, Z_{0}^{\prime}\right) \in \mathcal{G} \mathcal{Q}$, where $e \in E_{r}$ is adjacent to $v_{0}$. If $i x_{0}, i y_{0}$ or $z_{0} \in(-2,2)$, then $N=0$ and we are done (case (b) above). If $|z|,\left|z^{\prime}\right| \geq 2$ and both $z_{0}, z_{0}^{\prime}$ have the same signs then $N=0$ if $\left|z_{0}\right| \leq\left|z_{0}^{\prime}\right|$, and $N=1$ if $\left|z_{0}\right|>\left|z_{0}^{\prime}\right|$, where $v_{1} \leftrightarrow\left(X_{0}, Y_{0}, Z_{0}^{\prime}\right)$. By the results of [5] and [11, $v_{N}$ is a sink which is an attractor for the flow $f_{\rho}$ (see also the properties listed below), and we are done (case (a) above). If none of the above is satisfied, we perform the inductive step below.

Inductive step: Fix $k$ and suppose $v_{k} \leftrightarrow\left(X_{k}, Y_{k}, Z_{k}\right)$ and $\left|z_{k}\right| \geq 2$. Let $X_{k, n}, Y_{k, n}$, $n \in \mathbb{Z}$ be the successive neighbors of $Z_{k}$, where $X_{k}=X_{k, 0}$ and $Y_{k}=Y_{k, 0}$. Let $v_{k, 2 n} \leftrightarrow\left(Z_{k}, X_{k, n}, Y_{k, n}\right) \in \mathcal{G} \mathcal{T}$ and $v_{k, 2 n+1} \in V(\Sigma) \leftrightarrow\left(Z_{k}, Y_{k, n}, X_{k, n+1}\right)$ so that $\left\{v_{k, n}\right\}_{n \in \mathbb{Z}}$ are the successive vertices along $\partial Z_{k}$. Let $\varepsilon_{k, n} \in E_{r}$ be the edge with endpoint at $v_{k, n}$ which is not adjacent to $Z_{k}$ (see Figure 5). Using the sign change automorphism, we may assume that $z_{k} \geq 2$. Then $\left(x_{k, n}, y_{k, n}\right)$ and $\left(x_{k, n}, y_{k, n-1}\right)$ are coordinates of points on the hyperbola (two parallel lines if $z_{k}=2$ )

$$
-x^{2}-y^{2}+z_{k}(x y)+z_{k}^{2}=\kappa+2 .
$$

The successive values of $x_{k, n}, y_{k, n}$ in either direction can be obtained by looking at the intercepts of the up/down left/right zigzag path with the hyperbola (15), starting from the point $\left(x_{0}, y_{0}\right)=\left(x_{k, 0}, y_{k, 0}\right)$ (see Figure 6). We have the following facts:

(i) $x_{k, n}, y_{k, n} \rightarrow \pm \infty$ as $n \rightarrow \pm \infty$.

(ii) Either exactly one of $x_{k, n}, y_{k, n}=0$, or there is a unique vertex $v_{k, \min }$ adjacent to $Z_{k}$ at which there is a change of signs in successive values of $x_{k, n}, y_{k, n}$, that is, the other two regions adjacent to $v_{k, \text { min }}$ have purely imaginary values with different signs.

(iii) If $x_{k, n}, y_{k, n} \neq 0$ for all $n$, then $\tau\left(v_{k, n}\right)>\tau\left(v_{k, \min }\right)$ with strict inequality, for all $v_{k, n} \neq v_{k, \min }$. The same holds if exactly one of $x_{k, n}, y_{k, n}=0$, except now $\tau$ achieves its minimum on the two vertices on $\partial Z_{k}$ which are adjacent to the region with value 0 .

(iv) If $\left\{e_{k, n}\right\}_{n \in \mathbb{Z}}$ are the successive edges of $\partial Z_{k}$, then $f_{\rho}\left(e_{k, n}\right)$ is directed towards $v_{k, \min }$ for all $n \in \mathbb{Z}$, or the region $X_{k, l}$ (or $Y_{k, l}$ ) neighboring $Z_{k}$ with value 0 (in this case there is ambiguity in the definition of $f_{\rho}$ for the edge between $Z_{k}$ and the region $X_{k, l}$ (or $Y_{k, l}$ ) with value 0 ). This also follows from Proposition 3.3

Now starting at $v_{k}$, define a sequence of successive vertices along $\partial Z_{k}$ until by (ii) above, we either reach a vertex which has an adjacent region with value zero, or 


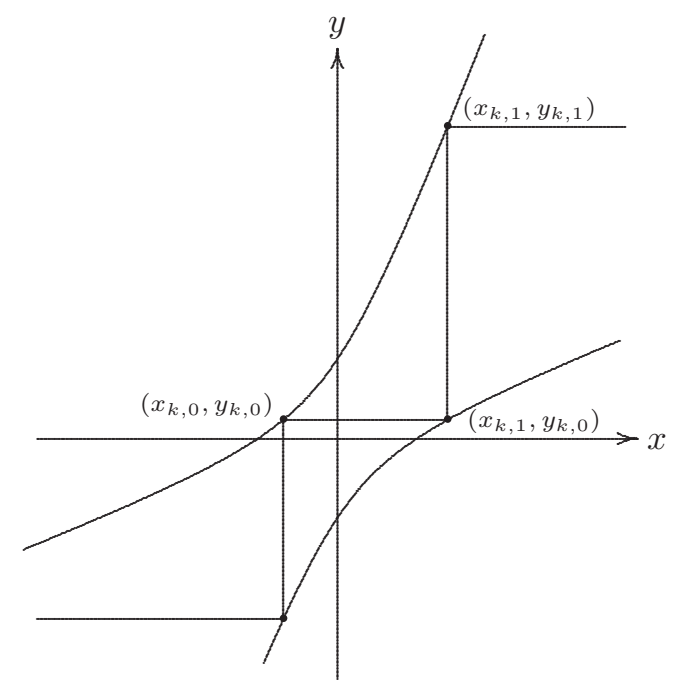

Figure 6 . The hyperbola $-x^{2}-y^{2}+z_{k}(x y)+z_{k}^{2}=\kappa+2\left(z_{k}=\right.$ $3, \kappa=0)$ and successive values of $x_{k, n}, y_{k, n}$.

we reach $v_{k, \min }$. In the first case, we end the algorithm at that point as we are in case (b). In the second case, if $\left(X_{k, n}, Y_{k, n}, Z_{k}, Z_{k}^{\prime}\right) \leftrightarrow \varepsilon_{k, m i n}$, we check the value of $z_{k}^{\prime}=-x_{k, n} y_{k, n}-z_{k}$. Note that $x_{k, n}$ and $y_{k, n}$ have different signs, so $-x_{k, n} y_{k, n}>0$, and that $z_{k}>2$ by assumption. If $z_{k}^{\prime} \geq z_{k} \geq 2$, we stop, as we are then in case (a); otherwise, we perform the second type of move and the next vertex is $v_{k, \text { min }}^{\prime}$ which is adjacent to $v_{k, \min }$ along $\varepsilon_{k, \min }$. If $z_{k}^{\prime} \geq 2$, we stop as again, we are in case (a). Similarly, if $z_{k}^{\prime} \in(-2,2)$, we stop, since we are in case (b). Finally, if $z_{k}^{\prime} \leq-2$, then $\left|z_{k}^{\prime}\right|<\left|z_{k}\right|$. We now proceed inductively with the new vertex $v_{k, \text { min }}^{\prime}$ taking the place of $v_{k}$, where we first perform a sign-change automorphism so we are back to the situation of $v_{k}$. It is clear that the algorithm does not backtrack. To show that it terminates after a number of steps, we need to consider two cases. If $|X|,|Y|$ is bounded away from 0 for all $X \in \mathcal{B}$ and $Y \in \mathcal{G}$, then for each step of the algorithm which is not a move along an edge of $E_{r}, \tau$ reduces by at least a fixed amount $K>0$ so the algorithm must terminate in a finite number of steps. Otherwise, there exists $\epsilon>0$ (depending only on $\kappa$ ) such that if $v_{k} \leftrightarrow\left(X_{k}, Y_{k}, Z_{k}\right)$ and $0<\left|i x_{k}\right|<\epsilon$, then $v_{k+1}, \cdots, v_{N}$ all lie on $\partial X_{k}$, and the algorithm terminates after a finite number of steps to case (b).

\section{REFERENCES}

[1] Brian H. Bowditch, Markoff triples and quasi-Fuchsian groups, Proc. London Math. Soc. (3) 77 (1998), no. 3, 697-736.

[2] William M. Goldman, Ergodic theory on moduli spaces, Ann. of Math. 146 (1997), 1-33.

[3] William M. Goldman, The modular group action on real SL(2)-characters of a one-holed torus, Geom. Topol. 7 (2003), 443-486.

[4] William M. Goldman, An exposition of results of Fricke, arXiv:math.GM/0402103

[5] William M. Goldman and George Stantchev, Dynamics of the automorphism group of the $G L(2, \mathbb{R})$-characters of a once-punctured torus, arXiv:math.DG/0309072 
[6] Linda Keen and Caroline Series, Pleating coordinates for the Maskit embedding of the Teichmüler space of punctured tori, Topology 32 (1993), no. 4, 719-749.

[7] Yair N. Minsky. The classification of punctured-torus groups, Ann. of Math. (2) 149 (1999), no. 2, 559-626.

[8] John A. G. Roberts, Escaping orbits in trace maps, Phys. A 228 (1996), no. 1-4, 295-325.

[9] Makoto Sakuma, Private communication, 2005.

[10] Ser Peow Tan, Yan Loi Wong and Ying Zhang, Necessary and sufficient conditions for McShane's identity and variations, arXiv:math.GT/0411184

[11] Ser Peow Tan, Yan Loi Wong and Ying Zhang, Generalized Markoff maps and McShane's identity, arXiv:math.GT/0502464

Department of Mathematics, National University of Singapore, 2 Science Drive 2, SiNGAPORE 117543

E-mail address: mattansp@nus.edu.sg

Department of Mathematics, National University of Singapore, 2 Science Drive 2, SiNGAPORE 117543

E-mail address: matwyl@nus.edu.sg

Department of Mathematics, Yangzhou University, Yangzhou, Jiangsu 225002, China

E-mail address: yingzhang@yzu.edu.cn 